\title{
INFLUENCE OF STRESS HISTORY ON UNDRAINED CYCLIC SHEAR STRENGTH EVOLUTION
}

\section{Fauzan Sahdi ${ }^{1 \& 2}$, Joe Tom $^{3}$, Zhechen Hou'2, Fraser Bransby', Christophe Gaudin $^{2}$ and Phillip Watson ${ }^{2}$}

\author{
${ }^{1}$ Civil Engineering Department, Faculty of Engineering, Universiti Malaysia Sarawak \\ ${ }^{2}$ Oceans Graduate School, The University of Western Australia \\ ${ }^{3}$ Civil and Environmental Engineering, University of Illinois at Urbana-Champaign \\ Corresponding Author: Zhechen Hou \\ Oceans Graduate School \\ The University of Western Australia \\ zhechen.hou@uwa.edu.au
}

Number of words: 6448 (excluding abstract, references, figures and tables)

Number of figures: 13

Number of tables: 2 


\begin{abstract}
Offshore infrastructure often interacts cyclically with the seabed over the operational life of a project. Previous research on the evolution of soil's undrained strength under long term, largeamplitude cyclic loading has focused on contractile clays and demonstrated that this cyclic interaction can lead to the initial generation and later dissipation of positive excess pore pressure in the soil. This process generally leads to an initial strength reduction, with subsequent densification and soil strength gains that can have consequences on the performance of seabed infrastructure during its design life. In this paper, new experimental data from T-bar penetrometer testing in kaolin and reconstituted Gulf of Mexico clays is presented. The data illustrate how the stress history, quantified via the overconsolidation ratio, affects soil strength changes during large-amplitude cyclic loading. The experiments explore both long-term continuous loading cycles and episodic loading with packets of undrained cycles followed by quiescent consolidation periods. A critical state-based framework is used to interpret the experimental data and provide predictions of the long-term steady-state strength of both soils as a function of the initial in situ state of the soil.
\end{abstract}

Key words: Cyclic loading, consolidation, overconsolidation ratio, T-bar penetrometer, undrained strength

\title{
INTRODUCTION
}

Offshore infrastructure, such as risers, well conductors, anchors and shallow foundations supporting pipeline infrastructure (Figure 1) are routinely subject to cyclic environmental and operational loads. Research in the past decades has shown that in normally consolidated to lightly overconsolidated (contractile) clays, the initial reduction in soil strength due to soil shearing can be eclipsed by soil strengthening due to reconsolidation during long-term loading, 
resulting in geo-structural capacity gains (e.g. Zdravković et al. 2003; Cocjin et al. 2017; Sahdi et al. 2017). For a system that can withstand short-term reductions in capacity, this strength evolution potentially provides an opportunity to take advantage of these long-term strength gains in design (Cocjin et al. 2017; Low et al. 2017; Abdel-Hakim et al. 2018). In contrast, strength (and stiffness) gains can also reduce structural system fatigue life and therefore need to be considered for robust infrastructure design (e.g. Clukey et al. 2017; Zhou et al. 2019a).

Recent research (Clukey et al. 2017; Cocjin et al. 2017; Zhou et al. 2019a; Zhou et al. 2019b) has incorporated critical state-based frameworks to capture the changing soil strength due to undrained cyclic loading followed by densification due to excess pore pressure generation and subsequent dissipation. These frameworks are based predominantly on experimental results in normally consolidated and lightly overconsolidated (contractile) clays, in which cyclic loading and soil remoulding generally always lead to net positive excess pore pressures and hence volume reduction and associated strength increases upon full reconsolidation. Although some deep-water clays are formed by geologically recent pelagic deposition and are normally consolidated, many are not and will be 'overconsolidated', either through mechanical or diagenetic processes, particularly near the seabed.

Various environmental processes during a soil's geological history affect the mechanical behaviour of soil, including purely mechanical overconsolidation through removal of overburden pressure through submarine slide events (Al-Khafaji et al. 2003; Schroeder et al. 2006), historical glaciation (Boulton and Dobbie 1993) or erosion (Silva and Jordan 1984; Chaney and Fang 1986). The response of such soils under shear is governed by the overconsolidation ratio (OCR), defined as the ratio of the preconsolidation to the in situ stress. Alternatively, longer-term in situ processes acting on geologically normally consolidated soils, such as biological activity that causes deep-water clay crusts (e.g. Moore 1931; Silva and 
Jordan 1984; Chaney and Fang 1986; Meadows et al. 2000; Kuo and Bolton 2012) and soil aging effects may also cause soils to be apparently overconsolidated, since a vertical stress in excess of the in situ stress is required to overcome the soil structure before yielding occurs. In these clays, the ratio of the yield to in situ stresses or the yield stress ratio (YSR) often exceeds the OCR, and it is the YSR which controls the behaviour of such clays under shear (Cotecchia and Chandler 1997; Cotecchia and Chandler 2000; Kuo and Bolton 2014). This behaviour is shown, for example, in the direct shear tests by Kuo and Bolton (2014), where mechanically normally consolidated natural 'structured' Gulf of Guinea clay samples were shown to also behave in a dilatant, apparently overconsolidated, manner.

Consequently, across a variety of offshore environments, seabed soils may often not be contractile during (large amplitude) shear and may not generate positive excess pore pressures described by previous cyclic loading and reconsolidation models. Thus, the cyclic interaction of infrastructure with soils near the seabed surface (such as shown on Figure 1) may have significant long-term behaviour differences when compared to that for normally consolidated clays. In this paper, we limit our study to the cyclic reconsolidation behaviour of normally consolidated and mechanically overconsolidated clays. Based on similarities in the manner that mechanically and non-mechanically 'overconsolidated' clays respond under monotonic shearing, the general behavioural observations in response to soil remoulding and reconsolidation presented in this paper may be applicable to both conditions, although this requires further investigation.

Insights into the effects of soil stress history (quantified using the overconsolidation ratio OCR - given as the ratio of maximum previous vertical effective stress to current vertical effective stress) on capacity changes for offshore infrastructure under cyclic loading can be obtained from a recent study by Hou et al. (2021). They reported centrifuge tests investigating 
long-term pipe-soil vertical stiffness changes in kaolin clay with different OCRs ranging from $\sim 21$ close to the seabed to 1 deep below the mudline. They found that changes in pipe-soil vertical stiffness are strongly linked to the soil state (characterised by the sample void ratio, $e$, and vertical effective stress, $\sigma_{v}^{\prime}$ ) relative to the soil normal compression line and failure lines relevant for intact and remoulded soil strength conditions. However, the tests did not reach a clear steady-state plateau as achieved in previous tests in contractile soils reported by Cocjin et al. (2014) and Yuan et al. (2017); and uncertainty remains as to whether overconsolidated soils will continue to dilate (and presumably lose strength) towards a steady-state strength or if strength gain will eventuate under long-term cyclic loading.

Consequently, this paper investigates the effects of soil stress history or, more generally, soil state on strength evolution (as opposed to pipe-soil stiffness studied by Hou et al. (2021)) in reconstituted soils under long-term displacement controlled cyclic loading. Experiments conducted using a T-bar penetrometer to explore strength evolution are described and the results also have direct relevance to the design of cylindrically shaped infrastructure, such as pipelines, risers, piles and well conductors. The experiments also provide insight into how the capacity of other geosystems such as anchors and shallow foundations may change under prolonged cyclic loading for soils with a range of stress histories. Finally, the modified critical state-based framework originally proposed by Hou et al. (2021) is extended to aid interpretation of the test results.

\section{TESTING METHODOLOGY}

Five displacement-controlled T-bar tests were conducted in kaolin and reconstituted Gulf of Mexico (GoM) clays prepared at OCRs ranging from 1 to 200, using both centrifuge (i.e. enhanced gravity) and laboratory floor (i.e. unit gravity) conditions. Table 1 describes the 
geotechnical properties of both clays. Continuous long-term cyclic T-bar tests were conducted in the kaolin sample, whereas testing in the GoM clay samples involved episodic T-bar cycles with periods of reconsolidation between adjacent cyclic episodes.

\section{Soils Tested and Sample Preparation}

\section{Kaolin clay}

The tests in kaolin clay were conducted in the 1.8 m radius geotechnical beam centrifuge at The University of Western Australia (UWA) (Randolph et al. 1991) for OCR $=2$ and 10 . Further kaolin testing was performed on the laboratory floor (at unit gravity) for OCR $=200$. A test strongbox with inner dimensions of $390 \mathrm{~mm}$ (width) $\times 650 \mathrm{~mm}$ (length) $\times 325 \mathrm{~mm}$ (height) with two-way vertical drainage was used during all tests.

To prepare the sample, kaolin was mixed at a moisture content of $120 \%$ (twice its liquid limit) and poured into the strongbox. To achieve the intended OCR of 2 , the sample was first consolidated in-flight at $40 \mathrm{~g}$, before decreasing the g-level to $20 \mathrm{~g}$ and allowing pore pressures to equilibrate. T-bar testing was then conducted at $20 \mathrm{~g}$. After completion of the T-bar test, the sample was reconsolidated fully at $200 \mathrm{~g}$ before reducing the g-level to $20 \mathrm{~g}$ and allowing pore pressure to equilibrate to create a sample with $\mathrm{OCR}=10$, which was then followed by a second T-bar test. After completion of the OCR $=10$ centrifuge test, the sample was allowed to swell on the laboratory floor for two weeks to achieve OCR $=200$. A third T-bar test was then performed.

\section{Gulf of Mexico clay}

T-bar tests in GoM clay samples were all conducted at unit gravity using elevated vertical stresses applied via steel plates placed on top of the sample, which provided a constant overburden pressure throughout the tests. The plates were solid other than $30 \mathrm{~mm}$ diameter 
openings to allow for T-bar insertion. The GoM clay was sampled from a confidential project site (believed to be representative of general deep water GOM clay) and was reconstituted at a water content of $160 \%(\sim 1.6$ times the liquid limit $)$ in a large mechanical mixer for two days. The slurry was then poured into two strongboxes (with identical dimensions to those used for the kaolin sample), each with two-way drainage for sample consolidation.

The first GoM clay sample (Sample 'GoM1') was prepared in a normally consolidated state $(\mathrm{OCR}=1)$ by applying a stress of $10 \mathrm{kPa}$ directly to the top surface of the sample using steel plates and allowing the sample to consolidate. Approximately 30 days were required for the sample to achieve primary consolidation, which was ascertained through frequent sample height measurements.

The second GoM clay sample (Sample 'GoM2') was prepared to achieve OCR = 10 at the same $10 \mathrm{kPa}$ final applied vertical stress. To achieve this, the slurry in the second strongbox was first loaded to $100 \mathrm{kPa}$ in a hydraulic press. After primary consolidation was achieved at $100 \mathrm{kPa}$ ( 21 days), steel plates applying a stress of $10 \mathrm{kPa}$ were placed on the top surface of the sample and the sample was allowed to fully swell ( $\sim 14$ days as determined through sample height measurements).

\section{TEST PROGRAMME}

All tests were conducted using a $5 \mathrm{~mm}$ (diameter) $\times 20 \mathrm{~mm}$ (length) $\mathrm{T}$-bar penetrometer to measure the vertical cyclic soil resistance. The T-bar resistance is interpreted in terms of nominal undrained shear strength, which was obtained by dividing the pressure due to T-bar perturbation in soil by a nominal bearing factor $\left(N_{T-\text { bar }}\right)$ of 10.5 (Randolph and Houlsby 1984; Martin and Randolph 2006). A T-bar undrained strength correction for shallow sample depths accounting for potential near-surface shallow failure mechanisms (White et al. 2010) was not 
required because all the T-bar tests reported in this paper were cyclic and conducted deep within the clay samples (shallowest depth of $\sim 16$ T-bar diameters) where a full-flow mechanism is expected.

\section{Test programme in kaolin clay}

Table 2(a) presents the T-bar cyclic test programme in kaolin. Tests DC1-K and DC2-K were conducted in the centrifuge and test $\mathrm{DC} 3-\mathrm{K}$ was conducted on the laboratory floor. These tests were conducted under displacement-control (i.e. oscillating between two fixed positions below the soil surface) at a constant velocity of $1 \mathrm{~mm} / \mathrm{s}$ with a cyclic amplitude of $10 \mathrm{~mm}(2 \mathrm{~T}$-bar diameters, D) for a fixed number of T-bar cycles. The T-bar tests dimensionless velocities, $V=v D / c_{v}$ are also shown in Table 2(a), with the coefficient of consolidation, $c_{v}$ calculated as:

$$
c_{v}=\frac{k E_{o}}{\gamma_{w}}
$$

where $k$ is the soil permeability (see Table 1), $\gamma_{w}$ is the unit weight of water and $E_{o}$ is the soil stiffness under one-dimensional loading/unloading-reloading. $E_{o}$ can be taken as $(1+e) \sigma_{v}^{\prime} / \lambda$ or $(1+e) \sigma_{v}^{\prime} / \kappa$ for normally consolidated or overconsolidated samples, respectively. The values of $V$ for the tests suggest that the instantaneous T-bar pass induces an undrained response in the soil $(V>10$ is considered to be undrained as per Chung et al. (2006)).

\section{Test programme in reconstituted GoM clay}

The displacement-controlled T-bar test programme in GoM clay is presented in Table 2(b), comprising episodic cyclic T-bar tests at a constant velocity of $1 \mathrm{~mm} / \mathrm{s}$. In sample GoM1 $(\mathrm{OCR}=1)$, Test DC-GoM1 comprises of a total of five episodes, each with 20 penetration and extraction cycles with an amplitude of $10 \mathrm{~mm}$ (2D). The values of $V$ for each test are also given in Table 2(b), where the $c_{v}$ was calculated from equation (1) using soil parameters shown in Table 1(b). The same procedure was adopted for test DC-GoM2 in sample GoM2 (OCR = 10), 
but only three cyclic episodes were performed (Table 2(b)). For the tests in these two samples, after each cyclic episode, the T-bar was held stationary at the centre point of the cycles for 10 hours to allow for soil consolidation. Using $c_{v}$ values of $1 \mathrm{~m}^{2} /$ year for sample GoM1 and $0.53 \mathrm{~m}^{2} /$ year for sample GoM2 (calculated using soil parameters shown in Table 1(b)), this will be equivalent to non-dimensional consolidation times, $T$ (taken as $c_{v} t /(0.5 D)^{2} ; t-$ consolidation time) of $\sim 183$ and 97 respectively, which is equivalent to $>90 \%$ consolidation according to Osman and Randolph (2012).

\section{SOIL STATE FRAMEWORK TO INTERPRET CYCLIC STRENGTH}

\section{CHANGES}

Before presenting the test results, this section introduces the framework employed to characterise how the initial soil state (initial void ratio and effective stress) influences the strength changes in clay under long-term cyclic loading. The soil behaviour is interpreted in void ratio - effective stress space as in the critical state soil mechanics framework (Roscoe and Burland 1968; Schofield and Wroth 1968) and builds upon recent work by White and Hodder (2010) and Hodder et al. (2013) to include the 'remoulded state line' (RSL). The RSL represents a state towards which soil strength fully degrades under large strains with negligible pore pressure dissipation (and therefore negligible strength recovery). A similar approach was employed by Hou et al. (2021) who utilised these concepts to describe measured changes in soil-pipe stiffness during centrifuge tests of a model riser segment.

\section{General framework}

Figure 2(a) presents data from a previous centrifuge test programme in kaolin clay (Hou et al. 2021) simulating the response of a model riser undergoing continuous vertical cyclic displacement in the top $\sim 1 \mathrm{~m}$ of the seabed. The data is presented as the evolution of pipe-soil 
unloading stiffness, $k_{s e c-n}$ for any cycle normalised by the initial unloading stiffness, $k_{s e c-i n i}$, where $k_{\text {sec }}$ was calculated as the change in pipe resistance normalised by twice the cyclic amplitude (peak-to-trough cyclic displacement) during pipe extraction phases. For all samples, an initial period of remoulding causes a loss in strength over the first $\sim 100$ cycles. For the normally consolidated sample, there is then an evident hardening response due to reconsolidation after $\sim 100$ cycles. However, this hardening response is significantly smaller for the samples with larger OCRs. There is a clear influence of the initial soil state on the evolution of $k_{s e c} / k_{s e c-i n i}$ (and by implication, soil strength).

Based on the data presented in Figure 2(a), Hou et al. (2021) suggested that the soil behaviour due to cyclic shearing and reconsolidation can be described by the in situ soil state in terms of void ratio $(e)$ and vertical effective stress $\left(\sigma_{v}^{\prime}\right)$ relative to three lines governing soil compression and shearing (see Figure 2(b)):

a) The one-dimensional normal compression line $\left(\mathrm{K}_{\mathrm{o}} \mathrm{NCL}\right)$, which governs the soil volumetric state (voids ratio, $e$ ) during normal compression.

b) The intact state line (ISL), which describes the soil state at initial monotonic failure. This is equivalent to the traditionally defined critical state line (Schofield and Wroth 1968).

c) The remoulded state line (RSL), which defines combinations of $e$ and $\sigma_{v}^{\prime}$ when the soil has been fully remoulded due to large shear strain and pore pressure accumulation.

To locate the ISL in $e-\sigma_{v}^{\prime}$ space, Hou et al. (2021) assumed that the mean effective stress on the $\mathrm{K}_{\mathrm{o}} \mathrm{NCL}$ is double the value on the CSL at the same void ratio (as adopted in the Modified Cam Clay (MCC) model for isotropically consolidated soils) and that the position of the RSL is defined by the soil sensitivity with respect to the ISL. They postulated that soil states in Zone A (between the $\mathrm{K}_{\mathrm{o}} \mathrm{NCL}$ and ISL) and Zone $\mathrm{C}$ (left of the RSL) will respectively harden 
or soften after a period of cyclic remoulding, whereas soil states in Zone B (between the ISL and RSL) will harden, but to a lesser degree than soils in Zone A (see Figure 2(b)).

It is unclear how extended shearing cycles affect the ultimate soil strength in higher OCR clays since it is evident in Figure 2(a) that the number of cycles for all three model pipe tests were insufficient to reach steady-state conditions. In this paper, this knowledge gap will be filled by analysing the results from a series of long-term cyclic T-bar tests. Also, the assumption made by Hou et al. (2021) that the position of the ISL is defined by a MCC yield criterion is relaxed; enabling more flexible estimation of the ISL and RSL positions and hence calculation of $\sigma_{S}^{\prime}-I S L$ and $\sigma_{s-R S L}^{\prime}$ along the unload-reload line, URL (see Figure 2(b)), which defines the limiting soil states influencing the behaviour of soils under long-term cyclic loading. In this paper, the three zones in Figure 2(b) are renamed as the 'wet', 'intermediate' and 'dry' zones to represent the different initial soil states.

Assuming that the critical state soil parameters are obtained from triaxial and one-dimensional consolidation tests, it may be shown that $\sigma_{s-I S L}^{\prime}$ and $\sigma_{S-R S L}^{\prime}$ can be expressed as (detailed derivation is provided in Appendix):

$$
\begin{gathered}
\frac{\left(3-2 \sin \phi^{\prime}\right) O C R_{I S L}}{1+2\left(1-\sin \phi^{\prime}\right) O C R_{I S L}^{\sin \phi^{\prime}}}=\exp \left(\frac{N_{K o}-\Gamma_{I S L}}{\lambda-\kappa}\right) \\
\frac{\left(3-2 \sin \phi^{\prime}\right) O C R_{R S L}}{1+2\left(1-\sin \phi^{\prime}\right) O C R_{R S L}^{\sin \phi^{\prime}}}=\exp \left(\frac{\lambda \ln S_{t}+N_{K o}-\Gamma_{I S L}}{\lambda-\kappa}\right)
\end{gathered}
$$

where $O C R_{I S L}=\sigma_{v c}^{\prime} / \sigma_{S-I S L}^{\prime}$ and $O C R_{R S L}=\sigma_{v c}^{\prime} / \sigma_{S-R S L}^{\prime}$ are the overconsolidation ratios for the soil stress states on the ISL and RSL respectively after swelling from the preconsolidation 
pressure, $\sigma_{v c}^{\prime}, S_{t}$ is the soil sensitivity, $\phi^{\prime}$ is the critical state friction angle, $\lambda$ and $\kappa$ are the slopes of the $\mathrm{K}_{\mathrm{o}} \mathrm{NCL}$, ISL or RSL and URL, respectively. $N_{K o}$ and $\Gamma_{I S L}$ are the void ratios at $p^{\prime}=1 \mathrm{kPa}$ on the $\mathrm{K}_{\mathrm{o}} \mathrm{NCL}$ and ISL, respectively. Although these functional forms require an iterative process to determine $O C R_{I S L}$ and $O C R_{R S L}$, they serve as useful parameters to determine the boundaries of the wet-intermediate and intermediate-dry zones as presented in Figure 2(b).

\section{STATE OF SOIL SAMPLES}

For the soil samples tested, using Equations (2) and (3) and the soil properties shown in Table 1 lead to $\mathrm{OCR}_{\mathrm{ISL}}$ and $\mathrm{OCR}_{\mathrm{RSL}}$ values as depicted in Figure 3. The OCRs for the tested kaolin (2, 10 and 200) and GoM clay (1 and 10) samples (denoted by grey circles) cover the wet, intermediate and dry zones. In the subsequent sections, the influence of the initial soil states (or soil stress histories) on the soil strength evolution under continuous and episodic cyclic Tbar tests is explored, where both the penetration and extraction T-bar data are presented.

\section{CYCLIC BEHAVIOUR OF SOIL IN THE WET ZONE $\left(\right.$ OCR $<$ OCR $\left._{\text {ISL }}\right)$}

Figure 4(a) shows the inferred nominal undrained shear strength $s_{u}$ throughout the whole of test DC1-K (kaolin, OCR $=2$; where $\mathrm{OCR}<\mathrm{OCR}_{\mathrm{ISL}}$ ) with selected cycles highlighted for comparison. In this figure (and in Figures 4(a), 5(a), 7(a), 9(a) and 10(a)), negative $s_{u}$ simply indicates that the T-bar is being extracted. Variation in $s_{u}$ with cycle number, $N$ is depicted in Figure 4(b), in terms of the ratio of strength at a given $N$ (in increments of 0.5 ) during both Tbar penetration and extraction $\left(s_{u-n}\right)$ to the initial $(\mathrm{N}=0.5)$ strength $s_{u-i n i}$ at the mid-cycle depth (approximately $z=100 \mathrm{~mm}$ ). For this test, $s_{u-n}$ at $N \sim 581$ (i.e. the final cycle) exceeds $S_{u-i n i}$ by $\sim 19 \%$. Unfortunately, T-bar test DCK-1 was prematurely terminated due to instrumentation error, and it is evident that the number of test cycles is insufficient to achieve 
steady-state $s_{u-s s} / s_{u-i n i}$ conditions (where $s_{u-s s}-$ steady-state strength), highlighting the remaining potential for soil hardening with further T-bar cycles. Consistent with the T-bar test results reported by Cocjin et al. (2014) for lightly-overconsolidated soils, the steady-state $s_{u-s s} / s_{u-i n i}$ would be expected to exceed unity.

The $s_{u}$ evolution for episodic T-bar tests in sample GoM1 with OCR = 1 (test DC1-GoM1; OCR $<\mathrm{OCR}_{\mathrm{ISL}}$ ) is shown in Figure 5. In this test, the T-bar was 'rested' at the mid-cycle depth (Figure 5(a)) for a period of 10 hours between cyclic episodes (20 cycles per episode) to allow for soil consolidation. This rest period is expected to correspond to $>90 \%$ consolidation as described in the 'Testing methodology' section. The results in Figure 5(a) indicate that $s_{u}$ upon re-penetration has an initial peak but reduces as the T-bar moves away from its resting position in each episode following consolidation. This reflects spatial variation in the strength changes that occur depending on the proximity of the soil to the T-bar resting position, presumably due to spatially varying total stress in the soil close to the T-bar. Figure 5(b) shows the variation in $s_{u-n} / s_{u-i n i}$ with $N$, where $s_{u-n}$ is measured once the T-bar moves $\sim 1 \mathrm{~mm}$ from the resting position, as shown in Figure 5(a). Figure 5(b) shows that in episodes $2-5$, there are subtle increases in both the re-penetration resistance and remoulded $s_{u}$.

Figure 6 depicts conceptual stress paths and $s_{u}$ evolution trends for both continuous and episodic T-bar tests interpreted from Figure 4 and Figure 5. Figure 6(a) presents the hypothesised stress path for continuous cyclic testing for soils in the wet zone with an initial mean effective stress $p_{W c 0}^{\prime}$. During the initial cyclic stages, negligible consolidation occurs and after initial failure at the ISL $\left(W_{c 1}\right)$, the soil state tends towards the RSL $\left(W_{c 2}\right)$. This is caused by the generation of net positive excess pore pressure, which reduces the operative soil strength from $s_{u W c 1}$ to $s_{u W c 2}$ as shown in Figure 6(b). As cycling progresses, consolidation effects increase and the alternating effects of shearing (which generates positive pore pressures) and 
consolidation (which involves pore pressure dissipation and thereby causes soil densification) result in the stress path approaching the in situ stress, $p_{W c 0}^{\prime}$, along the RSL at $W_{c 3}$ (see Figure 6(a)). At this stage, the soil reaches a steady-state strength, where no further excess pore pressure is generated from the T-bar cycles. This steady-state strength $\left(s_{u W c 3}\right.$ in Figure $\left.6(\mathrm{~b})\right)$ is larger than the initial strength $\left(s_{u W c 1}\right)$, even though it represents a 'remoulded' condition, because of the reduced void ratio. The qualitative description of the $e-p^{\prime}$ evolution here is consistent with T-bar test observations in Figure 4 (although the number or duration of T-bar cycles was insufficient to achieve a final steady-state strength) and previously reported penetrometer tests in kaolin with an initially 'wet' soil state (Cocjin et al. 2014).

The stress path for the episodic T-bar tests (Figure 6(c)) follows a similar general trend to the continuous test, except that the T-bar is halted to allow for longer periods of consolidation between relatively short periods of (predominantly undrained) cycling episodes. During cycling episodes, $p^{\prime}$ first reduces due to positive excess pore pressure generation from the in situ mean stress $\left(p_{W e 0}^{\prime}\right)$ to a soil state $W_{e 1}$ on the ISL and subsequently to $W_{e 2}$ on the RSL with additional cycles. This stress path is associated with an evolution of $s_{u}$ as schematically shown in Figure 6(d). If the soil fully consolidates back to $p_{W e 0}^{\prime}$ as the T-bar cycling is halted, the void ratio reduces by an amount governed by $\kappa$. T-bar cycles in the subsequent episode will cause the soil state to transition from $p_{W e 0}^{\prime}$ to $W_{e 3}$ and finally to $W_{e 4}$, where $p^{\prime}$ at $W_{e 3}>W_{e 1}$ and $W_{e 4}$ $>W_{e 2}($ Figure $6(\mathrm{c}))$. The re-penetration $\left(s_{u W e 3}\right)$ and remoulded $\left(s_{u W e 4}\right)$ soil strengths (see Figure 6(d)) are therefore higher than the respective strengths in the previous episode. If the number of episodes increases, it is hypothesised that the critical state and residual undrained shear strengths would continue to increase, and eventually be higher than the initial strength, similar to that observed in the continuous cycling tests. The qualitative description for episodic loading is broadly consistent with the episodic T-bar test results shown in Figure 5(b), except 
for the re-penetration $s_{u-n}$ in episode 2 being less than $s_{u-i n i}$ in episode 1. Similar behaviour was reported by Zhou et al. (2019b) who, from episodic T-bar tests in normally consolidated reconstituted carbonate soils, observed a reduction in the second episode of re-penetration $s_{u-n}$ compared to that in the first episode. The mechanics underlying this behaviour is not well understood but may be due to clay microstructure damage during the first cyclic episode, which cannot fully recover even after consolidation. Natural intact clays have structure which may be affected by long-term diagenetic process that will not be recovered during short-term (relative to a geological time scale) consolidation processes and, as such, may have less strength recovery. However, offshore infrastructure such as risers (see Figure 1) are continuously under cyclic motion, where any diagenetically formed clay structure will be destroyed in the first few cycles, implying that the design of such risers (governed by fatigue stresses) will depend predominantly on the soil states on the RSL. Nevertheless, attempts to account for the effect of soil structure in previous models have been implemented by translating the ISL to the left after the first cyclic episode (Cocjin et al. 2017; O'Loughlin et al. 2019).

\section{CYCLIC BEHAVIOUR OF SOIL IN THE INTERMEDIATE ZONE $\left(\mathrm{OCR}_{\mathrm{ISL}} \leq \mathrm{OCR} \leq \mathrm{OCR} \mathrm{RSL}\right)$}

Results from the continuous T-bar cyclic test in kaolin in the intermediate zone (test DC2-K; $\mathrm{OCR}=10 ; \mathrm{OCR}_{\mathrm{ISL}}<\mathrm{OCR}<\mathrm{OCR}_{\mathrm{RSL}}$ ) are shown in Figure 7. Similar to the continuous Tbar cyclic test in the wet zone (Figure 4 ), the operative $s_{u}$ decreases during the initial cycles before increasing for $N>100$. An apparent steady-state strength is then observed for $N>\sim 500$. In this case, the steady-state strength does not exceed the initial strength and instead tends to a steady-state strength ratio of $s_{u-s s} / s_{u-i n i} \sim 0.6$. 
Following the same reasoning as the previous section, we hypothesise a stress path to explain this strength evolution as depicted in Figure 8(a). For continuous T-bar cycling, $p^{\prime}$ transitions from the in situ value of $p_{I c 0}^{\prime}$ towards the ISL at $I_{c 1}$, generating negative excess pore pressure. With negligible consolidation during the first few cycles, $p^{\prime}$ then reduces from $I_{c 1}$ to $I_{c 2}$ on the RSL, 'erasing' the initial negative excess pore pressure. The strength correspondingly decreases from that at $s_{u I c 1}$ to $s_{u I c 2}$ as shown in Figure $8(\mathrm{~b})$. During subsequent alternating cycling and consolidation, the net positive excess pore pressure (albeit of smaller magnitude than in soils in the wet zone) dissipates and the soil state incrementally moves down the RSL, eventually reaching soil state $I_{c 3}$ at the in situ stress, $p_{I c 0}^{\prime}$. This reduction in void ratio is associated with a gradual increase in strength (Figure $8(\mathrm{~b})$ ). However, since $p_{I c 0}^{\prime}\left(\right.$ at $\left.I_{c 3}\right)$ is smaller than the value of $p^{\prime}$ on the ISL at $I_{c 1}$, the steady-state (remoulded) strength at $I_{c 3}\left(s_{u I c 3}\right)$ remains smaller than the initial strength, $s_{u I c 1}$.

\section{CYCLIC BEHAVIOUR OF SOIL IN THE DRY ZONE (OCR > OCR RSL $)$}

The continuous and episodic T-bar test results in kaolin (test DC3-K; OCR = 200) and GoM clay (test DC1-GoM2; OCR = 10) are presented in Figure 9 and Figure 10, respectively. These soil samples represent soil states in the dry zone, since $\mathrm{OCR}>\mathrm{OCR}_{\mathrm{RSL}}$. Based on both sets of results, soil consolidation (following cyclic loading) has the opposite effect than that in wet and intermediate soils, in that the $s_{u}$ generally reduces with increasing T-bar cycles (as shown in Figure 9) or post rest periods (as shown in Figure 10) with no apparent increases. Figure 11 schematically describes the evolution of soil state and undrained strengths for the continuous and episodic cyclic T-bar tests using the new framework.

For the continuous T-bar test, the stress path (Figure 11(a)) is similar to that for soils in the intermediate and the wet zones, except that in this case, negative excess pore pressures are 
predominantly generated since the stress path moves initially from the in situ stress, $p_{D c 0}^{\prime}$ to soil state $D_{c 1}$, and eventually to $D_{c 2}$, where net negative pore pressures remain (magnitude equal to $p_{D c 0}^{\prime}-D_{c 2}$ ), despite some reduction due to soil remoulding ( $D_{c 1}$ to $\left.D_{c 2}\right)$. The net negative excess pore pressures generated during undrained cycling means that upon dissipation, the soil swells (i.e. $e$ increases), causing a reduction in soil strength. This continuous alternating shearing and reconsolidation process eventually leads to a steady-state at $D_{c 3}$ at the in situ stress $p_{D c 0}^{\prime}$. This response presents a significantly different outcome to that in the other two zones with consolidation leading to a loss in strength as opposed to a gain (Figure 11(b)). Similar frameworks have been proposed by White et al. (2012) and Low et al. (2017) to capture the shear stress changes during episodic axial pipe-soil interface shearing and consolidation in overconsolidated soils, although these previous frameworks do not capture the change in axial pipe-soil peak shearing resistance and might be applicable only for quasi-cyclic shearing events.

The hypothesised stress path for the episodic T-bar test is depicted in Figure 11(c). The stress path in the first episode $\left(p_{D e 0}^{\prime}-D_{e 1}-D_{e 2}\right)$ is generally the same as the stress path in the initial stages of the continuous T-bar test (stress path $p_{D c 0}^{\prime}-D_{c 1}-D_{c 2}-$ Figure 11(a)), causing strength reduction as shown in Figure 11(d). During the rest periods in between cyclic episodes, full consolidation causes the soil to swell to the in situ stress, $p_{D e 0}^{\prime}$, along the URL as net negative excess pore pressures dissipate. In the subsequent T-bar cyclic episode, the soil state will follow the path $p_{D e 0}^{\prime}-D_{e 3}-D_{e 4}\left(\right.$ as in Figure 11(c)) causing the soil re-penetration $\left(s_{u D e 3}\right.$ ) and remoulded strengths $\left(s_{u D e}\right)$ to decrease relative to that in the previous episode $\left(s_{u D e 1}\right.$ and $\left.s_{u D e 2}\right)$ - Figure 11(d). Figure 10(b) suggests that the reduction in the re-penetration strength during subsequent episodes is more significant than the reduction in the remoulded strength (i.e. the strength measured after $\sim 20$ cycles in each episode) than that suggested in Figure 11(c) 
and (d). This may be due to: (i) damage to soil microstructure after T-bar cycling (as described previously for the wet zone results) potentially causing a significant decrease in the repenetration peak strength; or (ii) the low $\kappa / \lambda$ ratio of the GoM sample, which implies a relatively smaller change in void ratio (and hence strength reduction) per consolidation episode.

\section{STEADY-STATE SOIL STRENGTH}

The results in the previous sections provide insights into the effects of soil stress history on the overall evolution of strength during post-failure cyclic loading. The framework based on these observations can be used to predict the steady-state soil strength $\left(s_{u-s s}\right)$ resulting from continuous cycling.

Equation (A1) provides a means to predict the initial soil strength, linked to initial monotonic penetration of a T-bar. It can then be assumed that $s_{u-s s}$ corresponds to the strength after many cycles with concurrent consolidation and increases or decreases of the soil effective stress, such that it eventually corresponds to the RSL at the in situ stress (see Figure 6(a), Figure 8(a) and Figure 11(a)). If it is assumed that the soil friction angle $\left(\phi^{\prime}\right)$ and hence $M$ remains unchanged on the ISL and RSL, the strength on the RSL can also be quantified using equation (A1) but substituting $p_{I S L}^{\prime}$ with the initial in situ mean effective stress, $p_{o}^{\prime}$ (equal to $p_{R S L}^{\prime}$ ). With these assumptions, the ratio of the steady-state to initial soil strengths, $s_{u-s s} / s_{u-i n i}$ can be described by:

$$
\frac{s_{u-s s}}{s_{u-i n i}}=\frac{p_{o}^{\prime}}{p_{I S L}^{\prime}}
$$

Following Wroth (1984), $p_{o}^{\prime} / p_{I S L}^{\prime}$ (and therefore $s_{u-s s} / s_{u-i n i}$ ) can be expressed as a function of the soil unloading ratios, $R_{I S L}=p_{c}^{\prime} / p_{s-I S L}^{\prime}$ and $R=p_{c}^{\prime} / p_{o}^{\prime}$ (where $p_{c}^{\prime}$ and $p_{s-I S L}^{\prime}$ are the 
mean effective stress at the URL-KoNCL and URL-ISL intersections respectively as elaborated in the Appendix) and the soil critical state parameters:

$$
\frac{s_{u-s s}}{s_{u-i n i}}=\frac{p_{o}^{\prime}}{p_{I S L}^{\prime}}=\left(\frac{R_{I S L}}{R}\right)^{\Lambda}
$$

where $\Lambda=1-\kappa / \lambda$. From equations (A9) and (A10), equation (5) can be rewritten as:

$$
\frac{s_{u-s s}}{s_{u-i n i}}=\left[\frac{1+2\left(1-\sin \phi^{\prime}\right) O C R^{\sin \phi^{\prime}}}{\left(3-2 \sin \phi^{\prime}\right) O C R} \exp \left(\frac{N_{K o-} \Gamma_{I S L}}{\lambda-\kappa}\right)\right]^{\Lambda}
$$

Figure 12 compares the ratio $s_{u-s s} / s_{u-i n i}$ measured from the continuous T-bar tests in kaolin with $\mathrm{OCR}=2,10$ and 200 to that predicted by equation (6). This comparison is supplemented with data from a previous T-bar test by Cocjin et al. (2014) in lightly overconsolidated kaolin clay. The prediction provided by equation (6) shows reasonable agreement with the measurements across this data set, especially for higher OCR, although as previously noted there is uncertainty associated with the eventual $s_{u-s s}$ of the kaolin sample at OCR=2. This relationship provides a rational basis for predicting the steady-state strength of clays under continuous, large-strain cyclic loading and the soil parameters (OCR, $\left.N_{K o}, \lambda, \kappa, \Gamma_{I S L}, \phi^{\prime}\right)$ required to predict the ratio $s_{u-s s} / s_{u-i n i}$ can be obtained from conventional oedometer and triaxial tests. It is acknowledged that additional $s_{u-s s} / s_{u-i n i}$ data would be needed in future studies, especially for natural clays, to further validate equation (6).

\section{CONCLUSIONS}

This paper has highlighted the strong influence of soil stress history (as quantified in terms of overconsolidation ratio, OCR), on undrained strength changes that occur around a T-bar penetrometer under large-displacement, long-duration cyclic loading events in clays. Tests 
were conducted in reconstituted kaolin and Gulf of Mexico clays in the geotechnical centrifuge and at unit gravity, with OCR values ranging from 1 to 200 .

The strength changes measured during displacement-controlled T-bar cycles ranged from eventual strengths that exceeded the initial intact strength by more than $\sim 1.2$ times to strengths that reduce to $\sim 0.16$ times the initial intact strength, depending on the initial in situ soil state (i.e. combinations of soil void ratio and mean effective stress) relative to the normal compression, intact state and remoulded state lines. A framework based on critical state soil mechanics was used to explain the observed strength changes by linking the strength gains or losses to dissipation of positive or negative excess pore pressures (respectively) and to the associated volume changes occurring during the T-bar cycles. Using experimental observations and theoretical inferences, an analytical expression was provided to estimate the steady-state (or final) soil strength as a function of strength and compressibility parameters and the soil stress history under large-strain cyclic loading concurrent with consolidation.

This work highlights the complex interplay between soil stress and density conditions, and the potential evolution of the soil strength under long-term large amplitude cyclic loading conditions. Although strength was found to increase due to cyclic loading and reconsolidation for normally consolidated clays, long-term strength reductions are observed in overconsolidated soils. This means that detailed geotechnical characterisation of project sites is instrumental in potentially unlocking the benefits gained through consideration of these strength changes, and conversely in determining whether onerous strength changes (either strength gains or losses) will also affect offshore infrastructure in the long-term.

Although the work presented in this paper does not account for the effect of natural clay structure, it provides a framework for practicing engineers and geotechnical researchers to interpret and predict how the undrained strength of soil can evolve in the presence of large 
amplitude cyclic loading. For offshore infrastructure such as risers, the constant cyclic motion of this structure means that the short term effect of natural brittle clay structure is of secondary importance and the influence of soil state on the soil remoulding and reconsolidation behaviour of reconstituted soils presented in this paper may still be applicable.

\section{ACKNOWLEDGEMENTS}

The first and second authors are grateful for the postdoctoral financial support provided by the ARC Industrial Transformation Research Hub for Offshore Floating Facilities, which is funded by the Australian Research Council, Woodside Energy, Shell, Bureau Veritas and Lloyds Register (Grant No. IH140100012). The first author also acknowledges the financial support received from Universiti Malaysia Sarawak. The fourth author acknowledges the support of Fugro, provided via the Fugro Chair in Geotechnics at UWA. The sixth author leads the Shell Chair in Offshore Engineering research team at The University of Western Australia, which is sponsored by Shell Australia. 


\section{REFERENCES}

Abdel-Hakim, M., Abdel Azeem, M., Bransby, M.F., Low, H.E., Clavaud, R., Bergkamp, B., and Kizhikkilod, J. 2018. Cost Savings for Subsea Pipelines Using Enhanced Pipe-Soil Interaction Assessment. In Abu Dhabi International Petroleum Exhibition \& Conference. Society of Petroleum Engineers, Abu Dhabi, UAE.

Al-Khafaji, Z.A., Young, A.G., DeGroff, W., and Humphrey, G.D. 2003. Geotechnical properties of the Sigsbee escarpment from deep soil borings. In Offshore Technology Conference. Offshore Technology Conference, Houston, Texas, U.S.A.

Boulton, G.S., and Dobbie, K.E. 1993. Consolidation of sediments by glaciers: relations between sediment geotechnics, soft-bed glacier dynamics and subglacial ground-water flow. Journal of Glaciology 39(131): 26-44. doi: https://doi.org/10.3189/S0022143000015690.

Burland, J.B., Rampello, S., Georgiannou, V.N., and Calabresi, G. 1996. A laboratory study of the strength of four stiff clays. Géotechnique 46(3): 491-514. doi: https://doi.org/10.1680/geot.1996.46.3.491.

Chaney, R.C., and Fang, H.Y. 1986. Static and dynamic properties of marine sediments: A state of the art. In Marine Geotechnology and Nearshore/Offshore Structures. ASTM STP 923. American Society for Testing and Materials. pp. 74-111.

Chung, S.F., Randolph, M.F., and Schneider, J.A. 2006. Effect of penetration rate on penetrometer resistance in clay. Journal of Geotechnical and Geoenvironmental Engineering, ASCE 132(9): 1188-1196.

Clukey, E.C., Aubeny, C.P., Zakeri, A., Randolph, M.F., Sharma, P.P., White, D.J., Sancio, R., and Cerkovnik, M. 2017. A perspective on the state of knowledge regarding soil-pipe interaction for SCR fatigue assessments. In Offshore Technology Conference. Offshore Technology Conference, Houston, Texas, USA.

Cocjin, M., L., Gourvenec, S.M., White, D.J., and Randolph, M.F. 2017. Theoretical framework for predicting the response of tolerably mobile subsea installations. Géotechnique 67(7): 608-620. doi: https://doi.org/10.1680/jgeot.16.P.137.

Cocjin, M.L., Gourvenec, S.M., White, D.J., and Randolph, M.F. 2014. Tolerably mobile subsea foundations - observations of performance. Géotechnique 64(11): 895 - 909.

Cotecchia, F., and Chandler, R.J. 1997. The influence of structure on the pre-failure behaviour of a natural clay. Géotechnique 47(3): 523-544. doi: https://doi.org/10.1680/geot.1997.47.3.523.

Cotecchia, F., and Chandler, R.J. 2000. A general framework for the mechanical behaviour of clays. 50(4): 431-447. doi: https://doi.org/10.1680/geot.2000.50.4.431. 
DeJong, J., Yafrate, N., DeGroot, D., Low, H.E., and Randolph, M.F. 2010. Recommended practice for full-flow penetrometer testing and analysis. ASTM Geotechnical Testing Journal 33(2): 137-149.

Einav, I., and Randolph, M.F. 2005. Combining upper bound and strain path methods for evaluating penetration resistance. International Journal For Numerical Methods In Engineering 63: 1991-2016. doi: https://doi.org/10.1002/nme.1350.

Hodder, M., White, D.J., and Cassidy, M.J. 2013. An effective stress framework for the variation in penetration resistance due to episodes of remoulding and reconsolidation. Géotechnique 63(1): 30 - 43. doi: https://doi.org/10.1680/geot.9.P.145.

Hou, Z., Sahdi, F., Gaudin, C., and Randolph, M.F. 2021. Centrifuge modelling of pipe-soil interaction in clay with crust layer. Marine Structures 75: 102876. doi: https://doi.org/10.1016/j.marstruc.2020.102876.

Jaky, J. 1948. Pressure in soils. In Proceedings of The Second International Conference on Soil Mechanics and Foundation Engineering, London. pp. 103-107.

Kuo, M., and Bolton, M.D. 2012. The nature and origin of deep ocean clay crust from the Gulf of Guinea. Géotechnique 63(6): 500-509. doi: https://doi.org/10.1680/geot.10.P.012.

Kuo, M., and Bolton, M. 2014. Shear tests on deep-ocean clay crust from the Gulf of Guinea. Géotechnique 64(4): 249-257. doi: http://dx.doi.org/10.1680/geot.13.P.020.

Lehane, B.M., O'Loughlin, C.D., Gaudin, C., and Randolph, M.F. 2009. Rate effects on penetrometer resistance in kaolin. Géotechnique 59(1): 41-52.

Low, H.E., Ramm, M., Bransby, M.F., White, D.J., and Westgate, Z.W. 2017. Effect of through-life changes in soil strength and axial pipe-seabed resistance for HPHT pipeline design. In Proceedings of the International Conference on Offshore Site Investigation and Geotechnics, SUT, London, London. pp. 841-849.

Lunne, T., Andersen, K.H., Low, H.E., Randolph, M.F., and Sjursen, M. 2011. Guidelines for offshore in situ testing and interpretation in deepwater soft clays. Canadian Geotechnical Journal 48(4): 543-556.

Martin, C.M., and Randolph, M.F. 2006. Upper-bound analysis of lateral pile capacity in cohesive soil. Geotechnique 56(2): 141-145.

Mayne, P.W., and Kulhawy, F.H. 1982. $\mathrm{K}_{\mathrm{o}}$ - OCR relationship in soil. Journal of the Geotechnical Engineering Division, ASCE 108(6): 851-872.

Meadows, A., Meadows, P.S., West, F.J.C., and Murray, J.M.H. 2000. Bioturbation, geochemistry and geotechnics of sediments affected by the oxygen minimum zone on the 
Oman continental slope and abyssal plain, Arabian Sea. Deep Sea Research Part II: Topical Studies in Oceanography 47(1-2): 259-280. doi: https://doi.org/10.1016/S09670645(99)00104-6.

Moore, H.B. 1931. The muds of the Clyde Sea area. III. Chemical and physical conditions; rate and nature of sedimentation; and fauna. Journal of the Marine Biological Association of the United Kingdom 17(2): 325-358. doi: https://doi.org/10.1017/S0025315400050876.

O'Loughlin, C.D., Zhou, Z., Stanier, S.A., and White, D.J. 2019. Load-controlled cyclic T-bar tests: a new method to assess the combined effects of cyclic loading and consolidation. Géotechnique Letters 9(4). doi: https://doi.org/10.1680/jgele.19.00030.

Osman, A.S., and Randolph, M.F. 2012. Analytical solution for the consolidation around a laterally loaded pile. International Journal of Geomechanics 12(3): 199-208. doi: https://doi.org/10.1061/(ASCE)GM.1943-5622.0000123.

Randolph, M.F., and Houlsby, G.T. 1984. Limiting pressure on a circular pile loaded laterally in cohesive soil. Geotechnique 34(4): 613-623.

Roscoe, K.H., and Burland, J.B. 1968. On the generalized stress-strain behaviour of wet clay. Engineering Plasticity.

Sahdi, F. 2013. The changing strength of clay and its application to offshore pipeline design. $\mathrm{PhD}$ thesis, The University of Western Australia, Australia.

Sahdi, F., White, D.J., and Gaudin, C. 2017. Experiments using a novel penetrometer to assess the changing strength of clay during remolding and reconsolidation. Journal of geotechnical and geoenvironmental engineering 143(4): 1 - 7.

Sahdi, F., Tom, J.G., Laham, N., Watson, P.G., Bransby, M.F., and Gaudin, C. 2021. Measurements of long-term strength changes due to cyclic loading in Gulf of Mexico clay. In 6th International Conference on Geotechnical and Geophysical Site Characterization. , Budapest, Hungary (accepted).

Schofield, A., and Wroth, P. 1968. Critical state soil mechanics. McGraw-hill.

Schroeder, K., Andersen, K.H., and Tjok, K.M. 2006. Laboratory testing and detailed geotechnical design of the Mad Dog Anchors. In Offshore Technology Conference. Offshore Technology Conference, Houston, Texas, U.S.A.

Silva, A.J., and Jordan, S.A. 1984. Consolidation properties and stress history of some deep sea sediments. In Seabed mechanics, Proceedings of IUTAM Symposium. Springer, United Kingdom. pp. 25-39. 
White, D.J., and Hodder, M. 2010. A simple model for the effect on soil strength of episodes of remoulding and reconsolidation. Canadian Geotechnical Journal 47(7): 821-826. doi: https://doi.org/10.1139/T09-137.

White, D.J., Gaudin, C., Boylan, N., and Zhou, H. 2010. Interpretation of T-bar penetrometer tests at shallow embedment and in very soft soils. Canadian Geotechnical Journal 47(2): 218229.

White, D.J., Campbell, M.E., Boylan, N.P., and Bransby, M.F. 2012. A new framework for axial pipe-soil interaction, illustrated by shear box tests on carbonate soils. In Offshore Site Investigation and Geotechnics: Integrated Technologies-Present and Future. Society for Underwater Technology, London. pp. 379-387.

Wroth, C. 1984. The interpretation of in situ soil tests. Géotechnique 34(4): 449-489.

Yuan, F., White, D.J., and O'Loughlin, C.D. 2017. The evolution of seabed stiffness during cyclic movement in a riser touchdown zone on soft clay. Géotechnique 67(2): 127-137. doi: https://doi.org/10.1680/jgeot.15.P.161.

Zdravković, L., Potts, D.M., and Jackson, C. 2003. Numerical study of the effect of preloading on undrained bearing capacity. International Journal of Geomechanics 3(1): 1-10. doi: https://doi.org/10.1061/(ASCE)1532-3641(2003)3:1(1).

Zhou, H., and Randolph, M.F. 2009. Resistance of full-flow penetrometers in rate-dependent and strain-softening clay. Géotechnique 59(2): 79-86. doi: https://doi.org/10.1680/geot.2007.00164.

Zhou, Z., O'Loughlin, C.D., and White, D.J. 2019a. An effective stress analysis for predicting the evolution of SCR-seabed stiffness accounting for consolidation. Géotechnique 70(5): 448467. doi: https://doi.org/10.1680/jgeot.18.P.313.

Zhou, Z., O'Loughlin, C.D., White, D.J., and Stanier, S.A. 2019b. Improvements in plate anchor capacity due to cyclic and maintained loads combined with consolidation. Géotechnique: 1-18. doi: https://doi.org/10.1680/jgeot.19.TI.028. 


\section{APPENDIX: DERIVATION OF EQUATIONS 2 AND 3}

First, it is assumed that the initial T-bar passage through a soil horizon corresponds to a soil stress state on the ISL ( $p_{I S L}$, as shown in Figure A1), such that the initial soil undrained strength $\left(s_{u-i n i}\right)$ is defined by:

$$
s_{u-i n i}=\frac{M}{2} p_{I S L}^{\prime}
$$

where $M$ is the soil frictional constant and a function of the soil critical state friction angle, $\phi^{\prime}$. It should be noted that during the first T-bar passage, the soil in the vicinity of the T-bar will be partially remoulded due to the accumulated high shear strains (Einav and Randolph 2005; Zhou and Randolph 2009) and so, $s_{u-i n i}$ predicted using equation (A1) can potentially be overestimated, especially for highly sensitive soils. Further T-bar cycles cause the soil state to tend towards $p_{R S L}^{\prime}$ (see Figure A1). Previously reported triaxial tests data suggests that $\phi^{\prime}$ at failure is the same in both intact and reconstituted natural clay samples (Burland et al. 1996; Cotecchia and Chandler 1997). By extension, it is assumed that $\phi^{\prime}$ and hence $M$ remain constant for soil states on the ISL and RSL. Therefore, the strength on the RSL can also be defined by equation (A1), by replacing $p_{I S L}^{\prime}$ with $p_{R S L}^{\prime}$. Hence, as in Hou et al. (2021) (albeit for onedimensional stress conditions), the soil sensitivity $\left(S_{t}\right)$ is defined as:

$$
S_{t}=\frac{s_{u-i n i}}{s_{u-r e m}}=\frac{p_{I S L}^{\prime}}{p_{R S L}^{\prime}}
$$

where $s_{u-r e m}$ is the remoulded strength as measured from T-bar cyclic tests after (typically) 10-20 cycles (DeJong et al. 2010; Lunne et al. 2011).

As can be seen in Figure A1, the $e$ for a given $p^{\prime}$ along the $\mathrm{K}_{\mathrm{o}} \mathrm{NCL}$ and ISL can be, respectively, defined as: 


$$
\begin{aligned}
& e_{K o}=N_{K o}-\lambda \ln p_{K o}^{\prime} \\
& e_{I S L}=\Gamma_{I S L}-\lambda \ln p_{I S L}^{\prime}
\end{aligned}
$$

where $e$ in both cases decreases with increasing $p^{\prime}$ as a function of a constant slope, $\lambda$ and values of $e$ at $p^{\prime}=1 \mathrm{kPa}$ for the $\mathrm{K}_{0} \operatorname{NCL}\left(N_{K o}\right)$ and ISL $\left(\Gamma_{I S L}\right)$. Similarly, the variation in void ratio, $e_{R S L}$ with $p_{R S L}^{\prime}$ along the RSL is defined by:

$$
e_{R S L}=\Gamma_{R S L}-\lambda \ln p_{R S L}^{\prime}
$$

where $\Gamma_{R S L}$ is the void ratio at $p^{\prime}=1 \mathrm{kPa}$ on the RSL. The RSL is assumed to have the same slope as the $\mathrm{K}_{\mathrm{o}} \mathrm{NCL}$ and ISL (i.e. $\lambda$ ). Utilising equation (A2) and considering the ISL slope, $\lambda$, the parameter $\Gamma_{R S L}$ anchoring the RSL in $e-p^{\prime}$ space from Figure A1 is:

$$
\Gamma_{R S L}=\Gamma_{I S L}-\lambda \ln S_{t}
$$

If $\Gamma_{I S L}$ is unknown (which is the case for the clay types used in this paper - see Table 1), it can be derived by considering undrained shearing of a normally consolidated soil. In this case, the soil state moves from the $\mathrm{K}_{\mathrm{o}} \mathrm{NCL}$ to the ISL under constant volume conditions. Equating $e$ predicted from equations (A3) and (A4) (as appropriate for undrained shearing) and combining equations (A1), (A3) and (A4) gives:

$$
\Gamma_{I S L}=N_{K o}+\lambda\left[\ln \left(\frac{2}{M}\right)+\ln \left(\frac{s_{u}}{p_{K o}^{\prime}}\right)_{n c}\right]
$$

where $\left(s_{u} / p_{K o}^{\prime}\right)_{n c}$ is the strength normalised by the mean effective stress under normally consolidated soil conditions $\left(p_{K o}^{\prime}\right)$ : 


$$
\left(\frac{s_{u}}{p_{K o}^{\prime}}\right)_{n c}=\frac{3 s_{u}}{\sigma_{v K o}^{\prime}+2 K_{o-n c} \sigma_{v K o}^{\prime}}=\left(\frac{3}{3-2 \sin \phi^{\prime}}\right)\left(\frac{s_{u}}{\sigma_{v K o}^{\prime}}\right)_{n c}
$$

where $K_{o-n c}$ is the normally consolidated earth pressure at rest, assumed to be $K_{o-n c}$ $=1-\sin \phi^{\prime}\left(\right.$ Jaky 1948), and $\sigma_{v K o}^{\prime}$ is the normally consolidated vertical effective stress.

From Equations (A7) and (A8), the parameter $\Gamma_{I S L}$ can be estimated from $\phi^{\prime}$ and the onedimensionally normally consolidated undrained strength ratio, $\left(s_{u} / \sigma_{v K o}^{\prime}\right)_{n c}$; of which the latter may be determined from T-bar $s_{u}$ measurements in soils of a known $\sigma_{v K o}^{\prime}$.

For a soil unloaded from a preconsolidation pressure, $p_{c}^{\prime}$ along the URL, the transitional stress states that divides the soil cyclic response into the wet, intermediate or dry zones, $p_{S}^{\prime}-I S L$ and $p_{S-R S L}^{\prime}$ can be determined by considering the slope of the $\mathrm{K}_{0} \mathrm{NCL}$ as $\lambda=a / b$ or $\lambda=c / d$ (see Figure A1). Some algebraic rearrangements of these expressions lead to representations of the transitional stresses $p_{S-I S L}^{\prime}$ and $p_{S-R S L}^{\prime}$ in normalised forms:

$$
\begin{gathered}
R_{I S L}=\operatorname{Exp}\left(\frac{N_{K o}-\Gamma_{I S L}}{\lambda-\kappa}\right) \\
R_{R S L}=\operatorname{Exp}\left(\frac{\lambda \ln S_{t}+N_{K o}-\Gamma_{I S L}}{\lambda-\kappa}\right)
\end{gathered}
$$

where $R_{I S L}=p_{c}^{\prime} / p_{s-I S L}^{\prime}$ and $R_{R S L}=p_{c}^{\prime} / p_{s-R S L}^{\prime}$

The unloading ratios $R_{I S L}$ and $R_{R S L}$ are somewhat analogous to the soil overconsolidation ratio (OCR), but since OCR is more commonly used in practice, it is beneficial to express $R_{I S L}$ and $R_{R S L}$ as functions of OCR and other soil properties. Assuming $K_{o-n c}$ and $K_{o-o c}$ for overconsolidated soils as $K_{o-n c}=1-\sin \phi^{\prime}\left(\right.$ Jaky 1948) and $K_{o-o c}=\left(1-\sin \phi^{\prime}\right) O C R^{\sin \phi^{\prime}}$ (Mayne and Kulhawy 1982), $R_{I S L}$ and $R_{R S L}$ may be related to OCR via: 


$$
\begin{gathered}
R_{I S L}=\frac{p_{c}^{\prime}}{p_{s-I S L}^{\prime}}=\frac{\sigma_{v c}^{\prime}+2 K_{o-n c} \sigma_{v c}^{\prime}}{\sigma_{s-I S L}^{\prime}+2 K_{o-o c} \sigma_{s-I S L}^{\prime}}=\frac{\left(3-2 \sin \phi^{\prime}\right) O C R_{I S L}}{1+2\left(1-\sin \phi^{\prime}\right) O C R_{I S L} \sin \phi^{\prime}} \\
R_{R S L}=\frac{p_{c}^{\prime}}{p_{S-R S L}^{\prime}}=\frac{\sigma_{v c}^{\prime}+2 K_{o-n c} \sigma_{v c}^{\prime}}{\sigma_{s-R S L}^{\prime}+2 K_{o-o c} \sigma_{S-R S L}^{\prime}}=\frac{\left(3-2 \sin \phi^{\prime}\right) O C R_{R S L}}{1+2\left(1-\sin \phi^{\prime}\right) O C R_{R S L} \sin \phi^{\prime}}
\end{gathered}
$$

Equation (A11) and (A12) and can be combined with equations (A9) and (A10) so that the OCR related to the stress states on the ISL $\left(O C R_{I S L}=\sigma_{v c}^{\prime} / \sigma_{s-I S L}^{\prime}\right)$ and RSL $\left(O C R_{R S L}=\sigma_{v c}^{\prime}\right)$ $\sigma_{s-R S L}^{\prime}$ ) can be expressed as equations (2) and (3) respectively. 
Table 1. Properties of: (a) kaolin; (b) Gulf of Mexico clay

\begin{tabular}{|c|c|c|}
\hline Parameters & Value & Reference \\
\hline Normal consolidation line (NCL) slope, $\lambda$ & 0.26 & \multirow{4}{*}{ Lehane et al. (2009) } \\
\hline Unload-reload line slope, $\kappa$ & 0.06 & \\
\hline Void ratio on $\mathrm{K}_{\mathrm{o}} \mathrm{NCL}$ at $p^{\prime}=1 \mathrm{kPa}, N_{K o}$ & 2.51 & \\
\hline$\phi^{\prime}$ & $26^{\circ}$ & \\
\hline T-bar strength ratio, $\left(s_{u} / \sigma_{v K o}^{\prime}\right)_{n c}$ & 0.15 & \multirow{3}{*}{ Sahdi (2013) } \\
\hline Sensitivity, $S_{t}$ & 2.5 & \\
\hline Permeability, $k(\mathrm{~m} / \mathrm{s})$ & $4 \times 10^{-10} e^{3.46}(e=$ void ratio $)$ & \\
\hline
\end{tabular}

(a)

\begin{tabular}{|c|c|c|}
\hline Parameters & Value & Reference \\
\hline Normal consolidation line (NCL) slope, $\lambda$ & 0.462 & \multirow{6}{*}{ Sahdi et al. (2021) } \\
\hline Unload-reload line slope, $\kappa$ & 0.049 & \\
\hline Void ratio on $\mathrm{K}_{\mathrm{o}} \mathrm{NCL}$ at $p^{\prime}=1 \mathrm{kPa}, N_{K o}$ & 3.74 & \\
\hline T-bar strength ratio, $\left(s_{u} / \sigma_{v K o}^{\prime}\right)_{n c}$ & 0.27 & \\
\hline Sensitivity, $S_{t}$ & 2 & \\
\hline Permeability, $k(\mathrm{~m} / \mathrm{s})$ & $2 \times 10^{-12} e^{8.27}(e=$ void ratio $)$ & \\
\hline$\phi^{\prime}$ & $30^{\circ}$ & $\begin{array}{c}\text { Regional } \\
\text { experience }\end{array}$ \\
\hline
\end{tabular}

(b) 
Table 2. Displacement control T-bar test parameters (Note: D - T-bar diameter): (a) continuous cyclic Tbar tests in kaolin clay; (b) episodic cyclic T-bar tests in Gulf of Mexico clay

\begin{tabular}{|c|c|c|c|c|c|c|}
\hline Test name & Sample & $\begin{array}{c}\text { OCR } \\
(-)\end{array}$ & $\begin{array}{c}\text { Cyclic } \\
\text { amplitude } \\
(\mathbf{m m})\end{array}$ & $\begin{array}{c}\text { Number of } \\
\text { cycles (-) }\end{array}$ & $\begin{array}{c}\text { Test } \\
\text { velocity } \\
(\mathrm{mm} / \mathrm{s})\end{array}$ & $\begin{array}{c}\text { Test non- } \\
\text { dimensional } \\
\text { velocity, } V\end{array}$ \\
\hline DC1-K & \multirow{3}{*}{ Kaolin } & 2 & $10(2 \mathrm{D})$ & 581 & \multirow{3}{*}{1} & 64 \\
\hline DC2-K & & 10 & $10(2 \mathrm{D})$ & 720 & & 111 \\
\hline DC3-K & & 200 & $10(2 \mathrm{D})$ & 1000 & & 2056 \\
\hline
\end{tabular}

(a)

\begin{tabular}{|c|c|c|c|c|c|c|c|}
\hline $\begin{array}{c}\text { Test } \\
\text { name }\end{array}$ & $\begin{array}{c}\text { Sample } \\
\text { name }\end{array}$ & $\begin{array}{c}\text { OCR } \\
(-)\end{array}$ & $\begin{array}{c}\text { Cyclic } \\
\text { amplitude } \\
(\mathbf{m m})\end{array}$ & $\begin{array}{c}\text { Number } \\
\text { of cycles } \\
\text { per } \\
\text { episode } \\
\text { (-) }\end{array}$ & $\begin{array}{c}\text { Number } \\
\text { of } \\
\text { episodes } \\
(-)\end{array}$ & $\begin{array}{c}\text { Test } \\
\text { velocity } \\
(\mathrm{mm} / \mathrm{s})\end{array}$ & $\begin{array}{c}\text { Test non- } \\
\text { dimensional } \\
\text { velocity, } V\end{array}$ \\
\hline $\begin{array}{c}\text { DC- } \\
\text { GoM1 }\end{array}$ & GoM1 & 1 & $10(2 \mathrm{D})$ & 20 & 5 & \multirow[t]{2}{*}{1} & 17 \\
\hline $\begin{array}{c}\text { DC- } \\
\text { GoM2 }\end{array}$ & GoM2 & 10 & $10(2 \mathrm{D})$ & 20 & 3 & & 259 \\
\hline
\end{tabular}

(b) 


\section{FIGURE CAPTIONS}

Figure 1. Examples of offshore infrastructure affected by cyclic loading

Figure 2. (a) Pipe-soil unloading stiffness (Hou et al. 2021); (b) Soil state framework in $e-\sigma_{v}^{\prime}$ space (adapted and modified from Hou et al. (2021))

Figure 3. Soil states for kaolin and Gulf of Mexico clay samples

Figure 4. Continuous cyclic T-bar test results in kaolin sample (test DC1-K; OCR $=2$ ), assuming $N_{T-\text { bar }}=10.5$ : (a) T-bar overall strength profile; (b) evolution of strength at the mid-cycle depth

Figure 5. Episodic cyclic T-bar tests in sample GoM1 (test DC-GoM1; OCR = 1), assuming $N_{T-\text { bar }}=10.5$ : (a) T-bar overall strength profile; (b) evolution of strength immediately below the T-bar resting location

Figure 6. Stress path and strength evolution for soils in the wet zone

Figure 7. Continuous cyclic T-bar test results in kaolin sample (test DC2-K; OCR $=10$ ), assuming $N_{T-\text { bar }}=10.5$ : (a) T-bar overall strength profile; (b) evolution of strength at the mid-cycle depth

Figure 8. Stress path and strength evolution for soils in the intermediate zone

Figure 9. Continuous cyclic T-bar test results in kaolin sample (test DC3-K; OCR = 200), assuming $N_{T-\text { bar }}=10.5$ : (a) T-bar overall strength profile; (b) evolution of strength at the mid-cycle depth 
Figure 10. Episodic cyclic T-bar tests in sample GoM2 (test DC-GoM2; OCR = 10), assuming $N_{T-\text { bar }}=10.5$ : (a) T-bar overall strength profile; (b) evolution of strength immediately below the T-bar resting location

Figure 11. Stress path and strength evolution for soils in the dry zone

Figure 12. Steady-state $s_{u-s s} / s_{u-i n}$ ratio

Figure A1. Soil state framework in $e-p^{\prime}$ space 


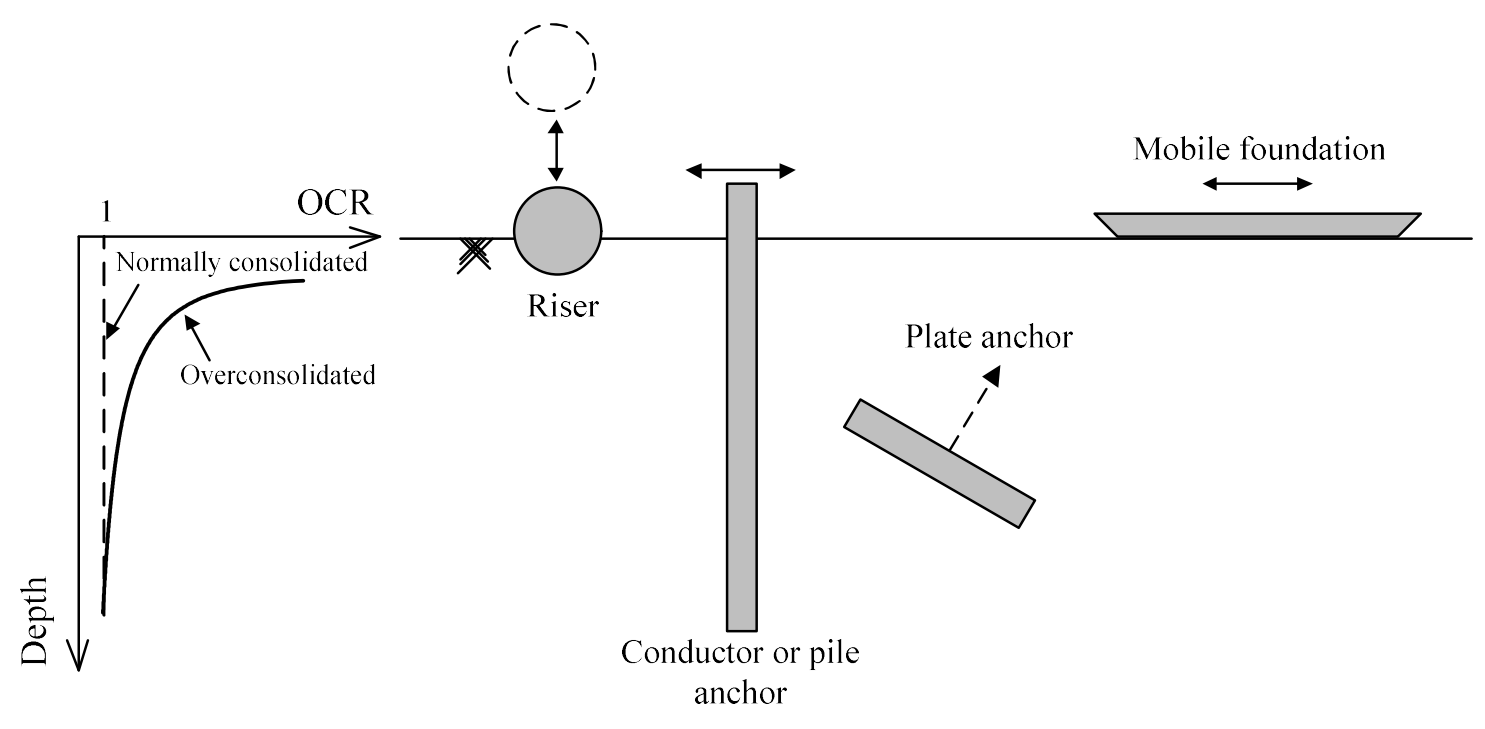

Figure 1. Examples of offshore infrastructure affected by cyclic loading 


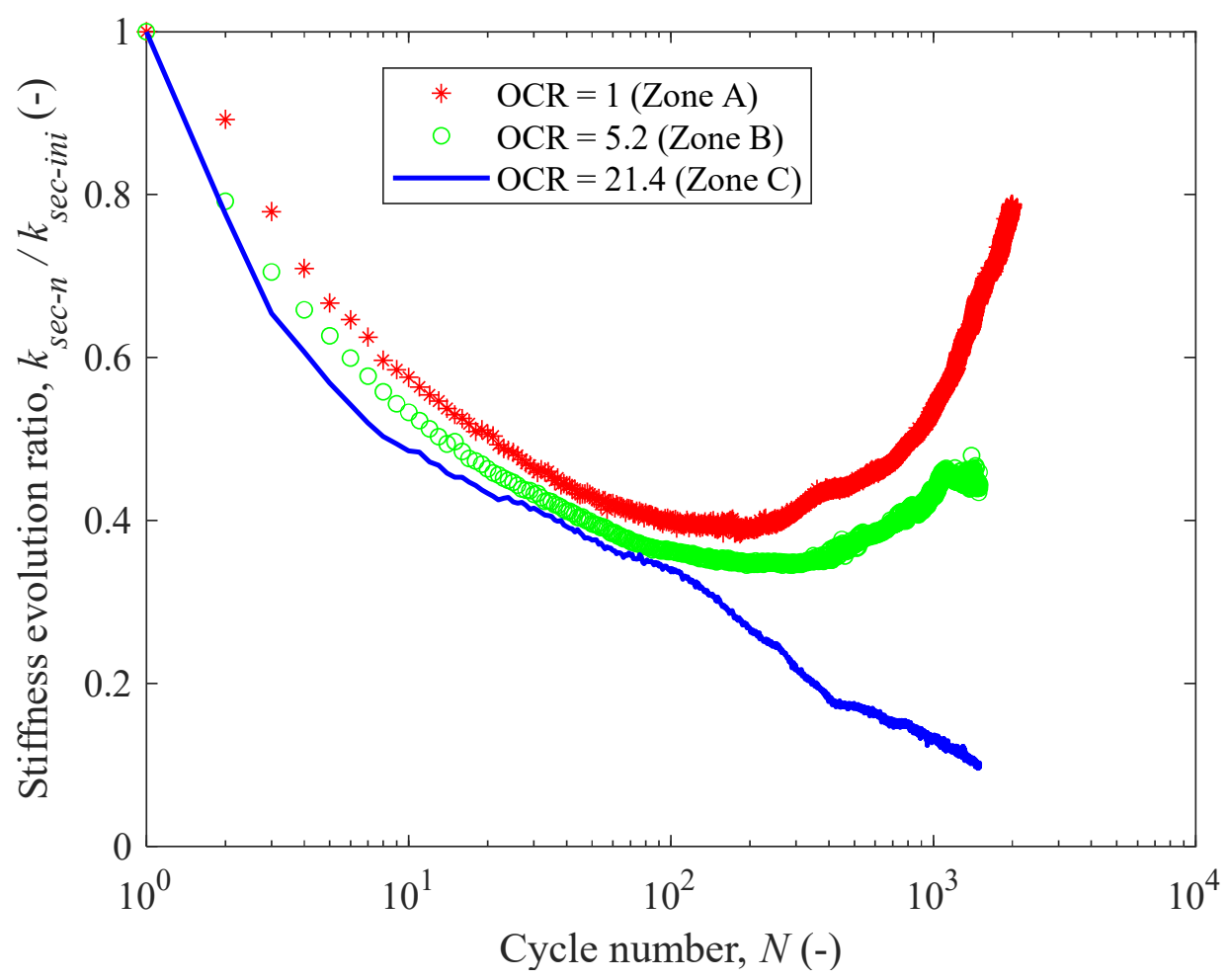

(a)

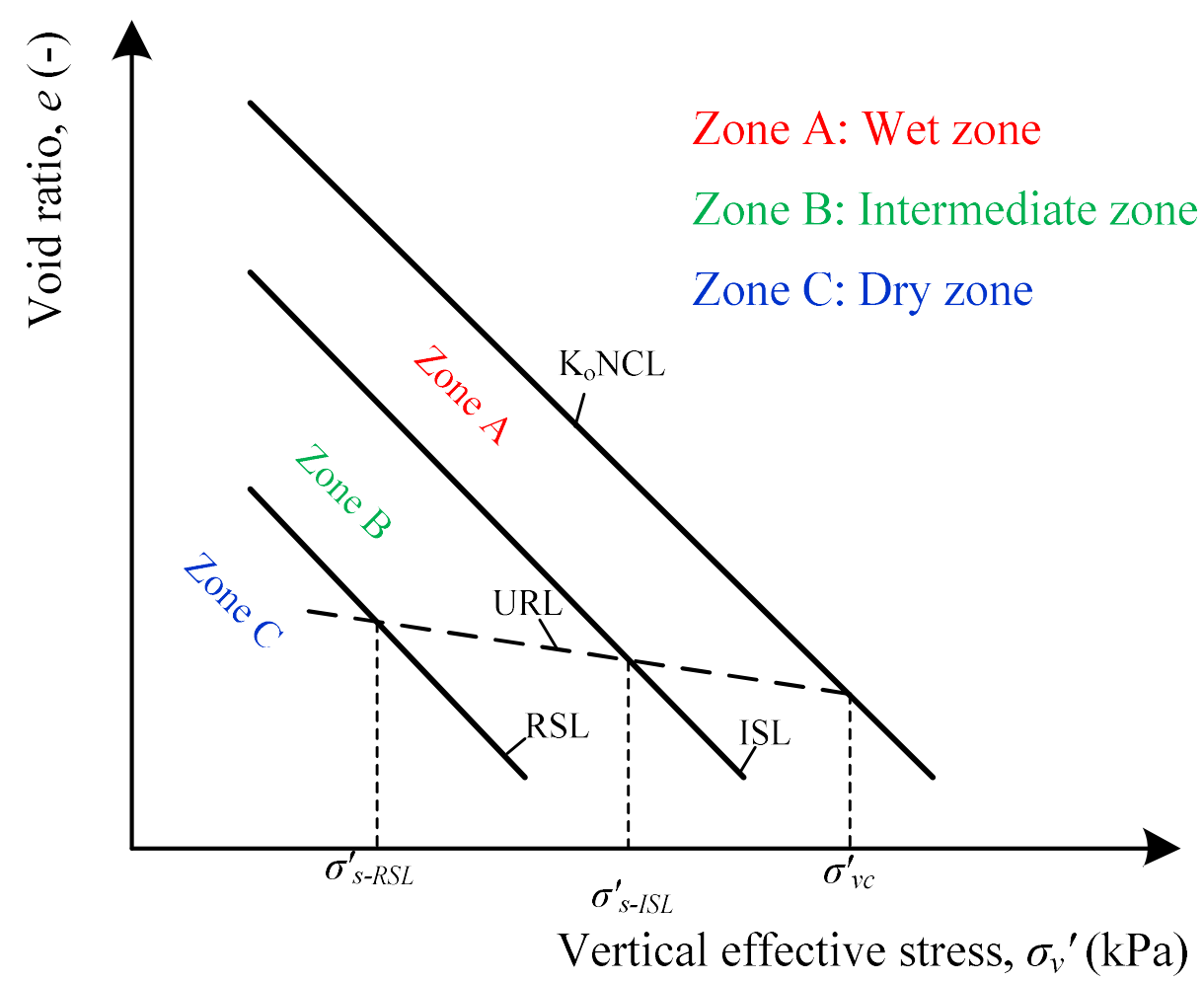

(b)

Figure 2. (a) Pipe-soil unloading stiffness (Hou et al. 2021); (b) Soil state framework in $e-\sigma_{v}^{\prime}$ space (adapted and modified from Hou et al. (2021)) 


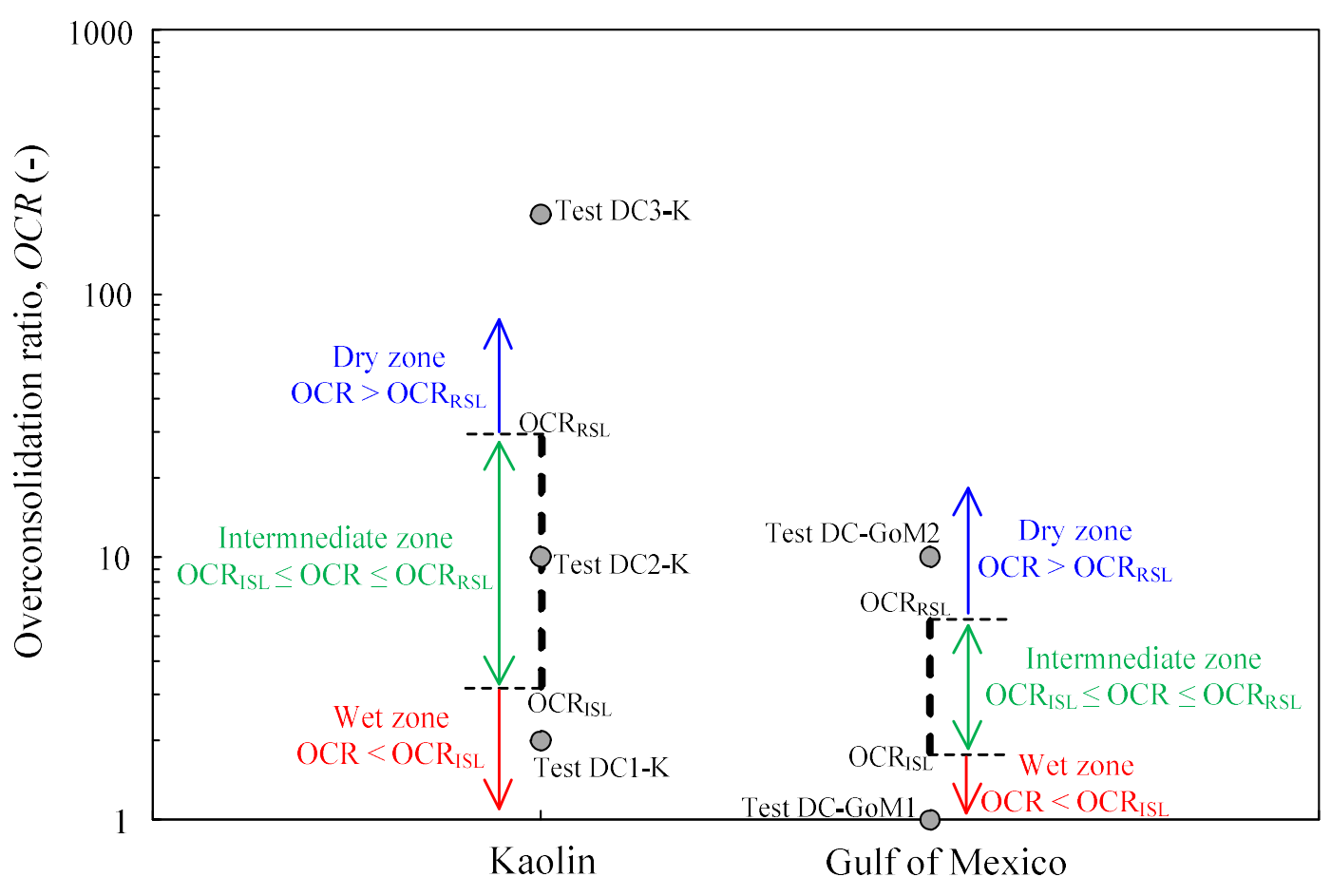

Figure 3. Soil states for kaolin and Gulf of Mexico clay samples 


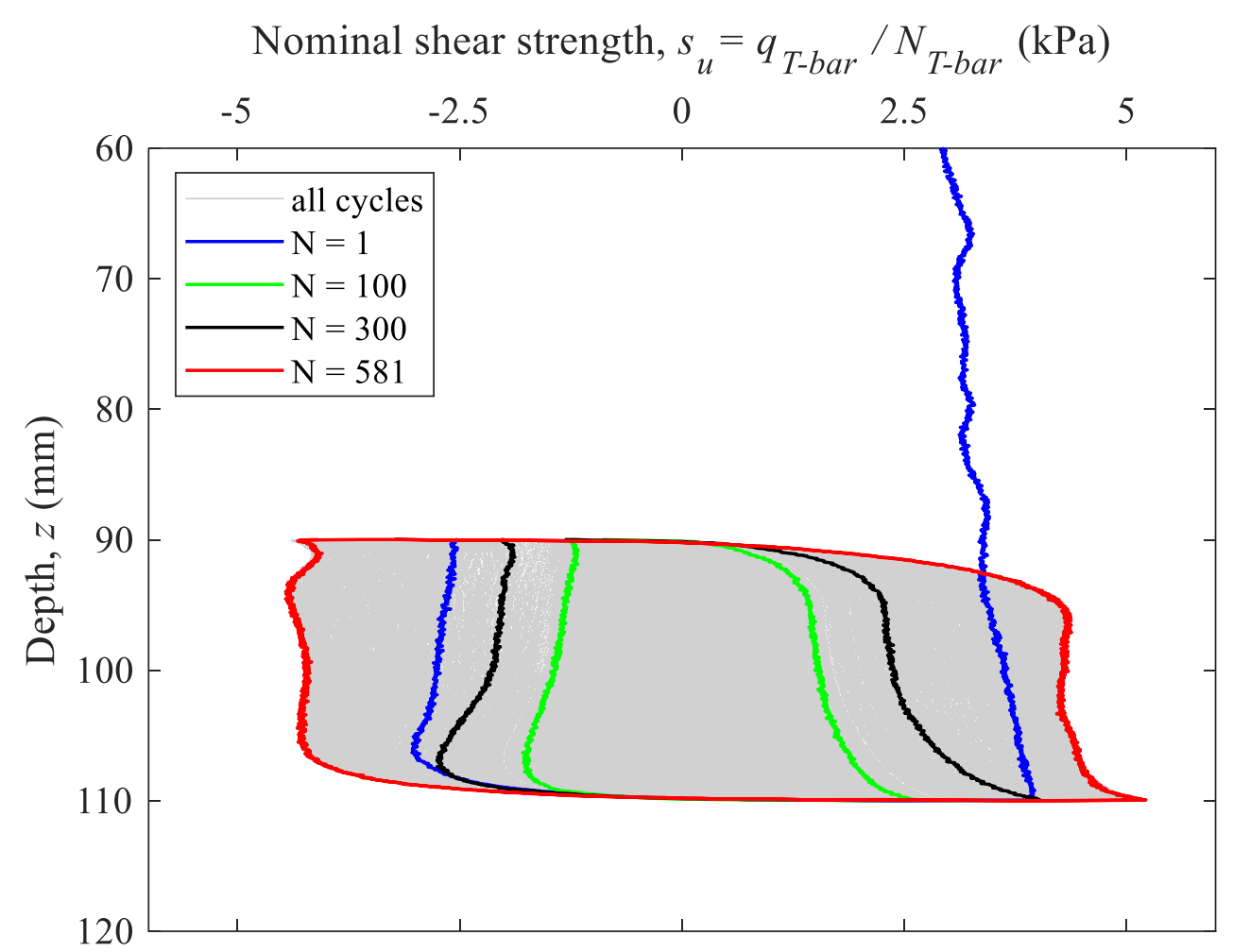

(a)

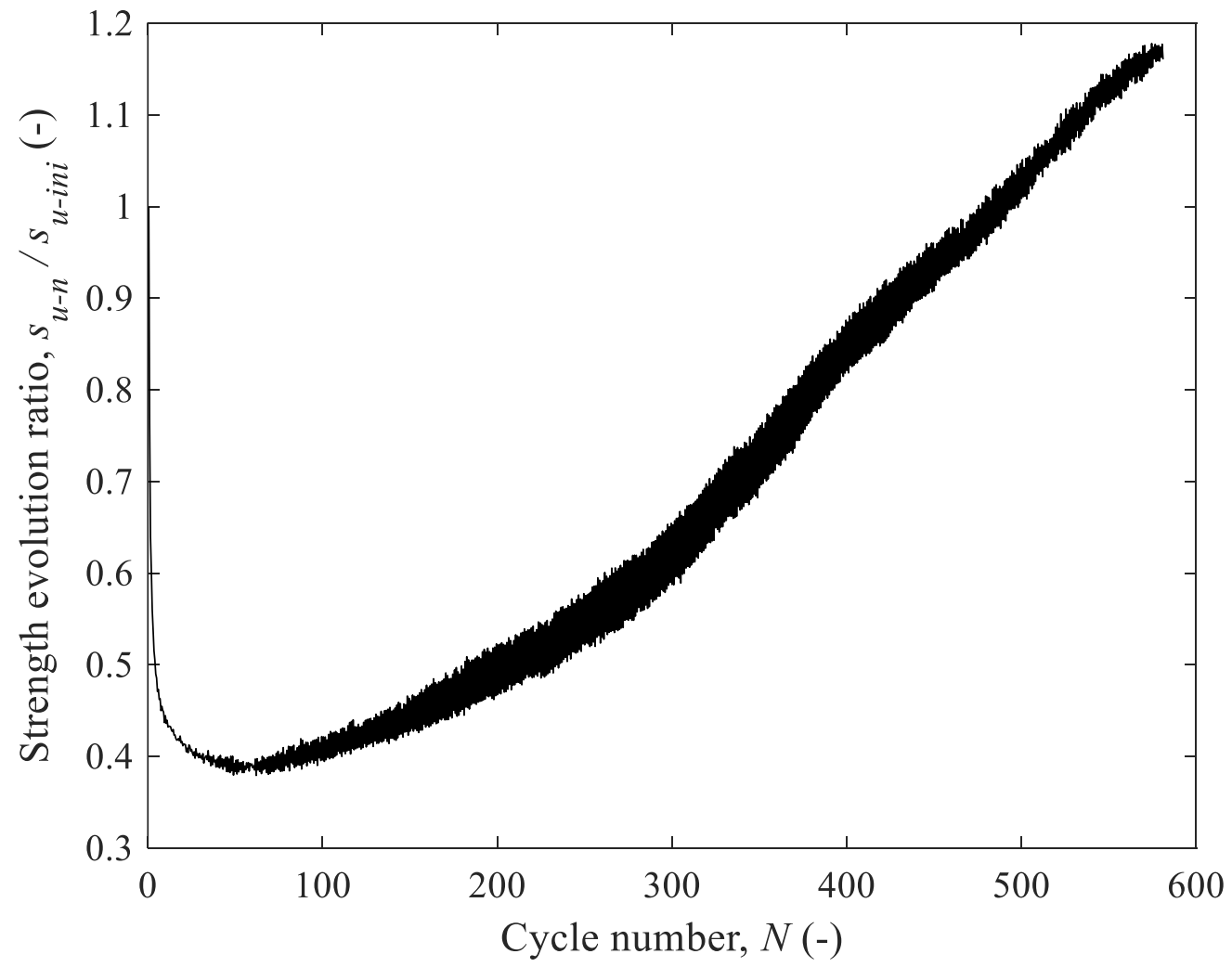

(b)

Figure 4. Continuous cyclic T-bar test results in kaolin sample (test DC1-K; OCR = 2), assuming $N_{T-b a r}=10.5$ : (a) T-bar overall strength profile; (b) evolution of strength at the mid-cycle depth 


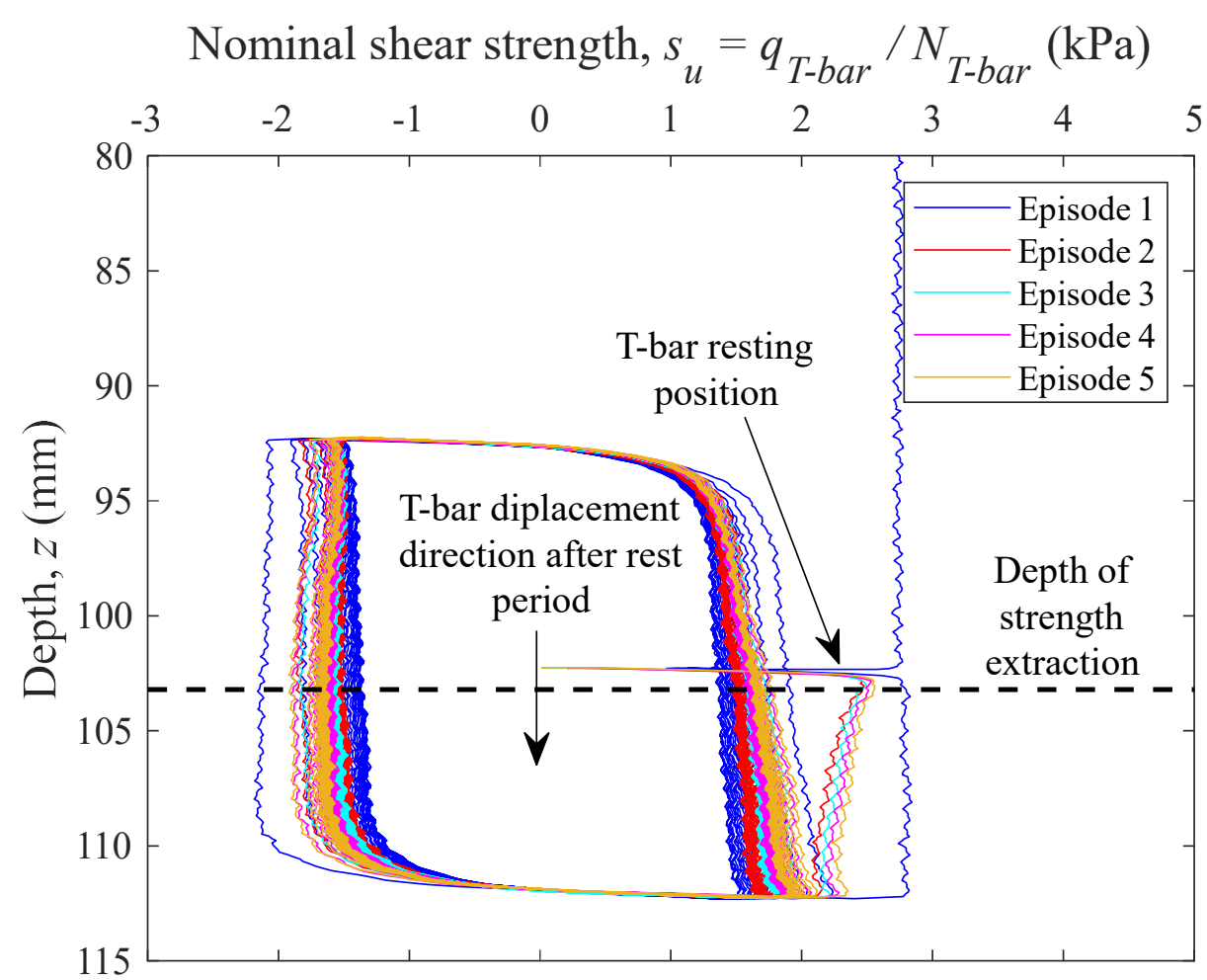

(a)

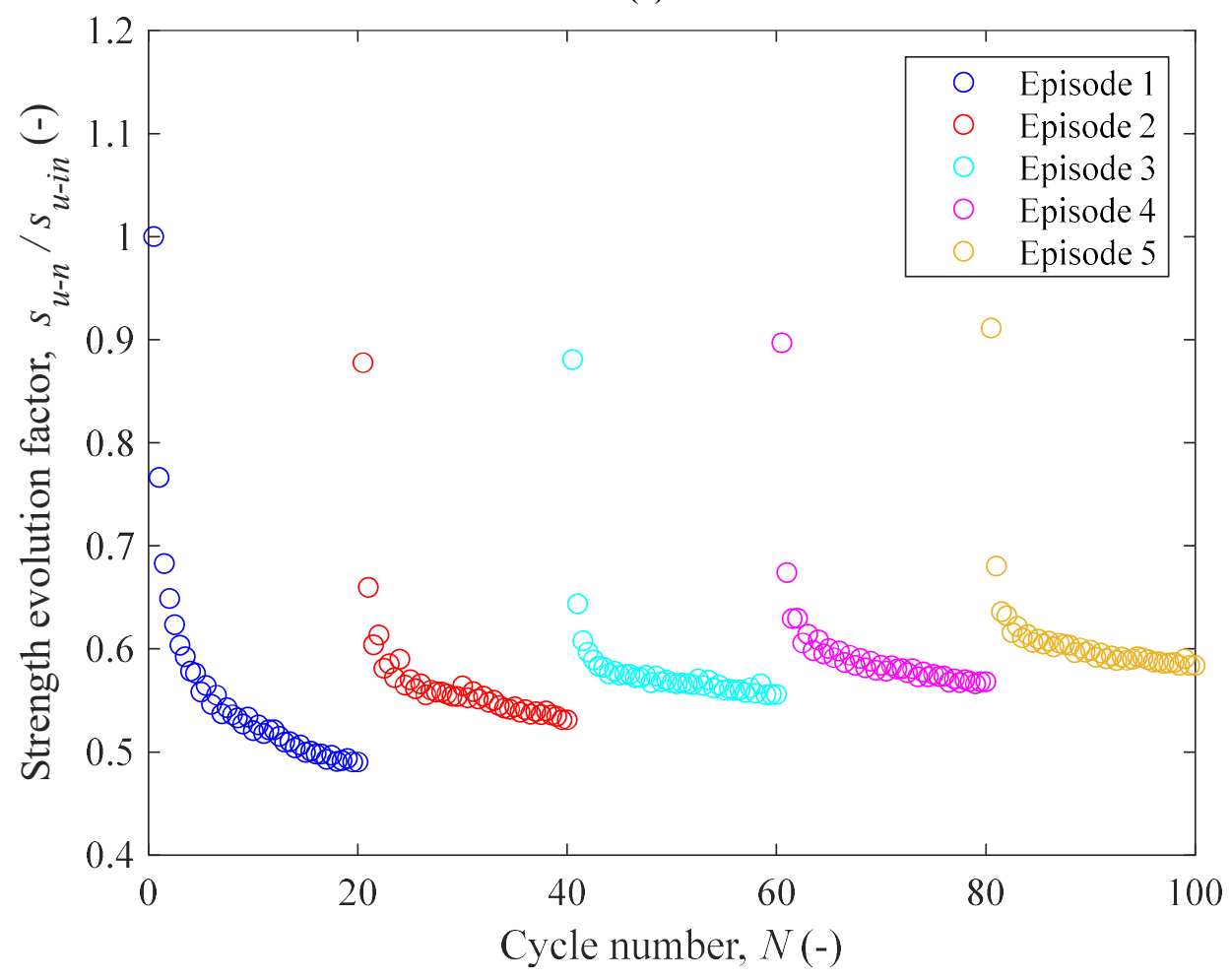

(b)

Figure 5. Episodic cyclic T-bar tests in sample GoM1 (test DC-GoM1; OCR = 1), assuming $N_{T-\text { bar }}=$ 10.5: (a) T-bar overall strength profile; (b) evolution of strength immediately below the T-bar resting location 


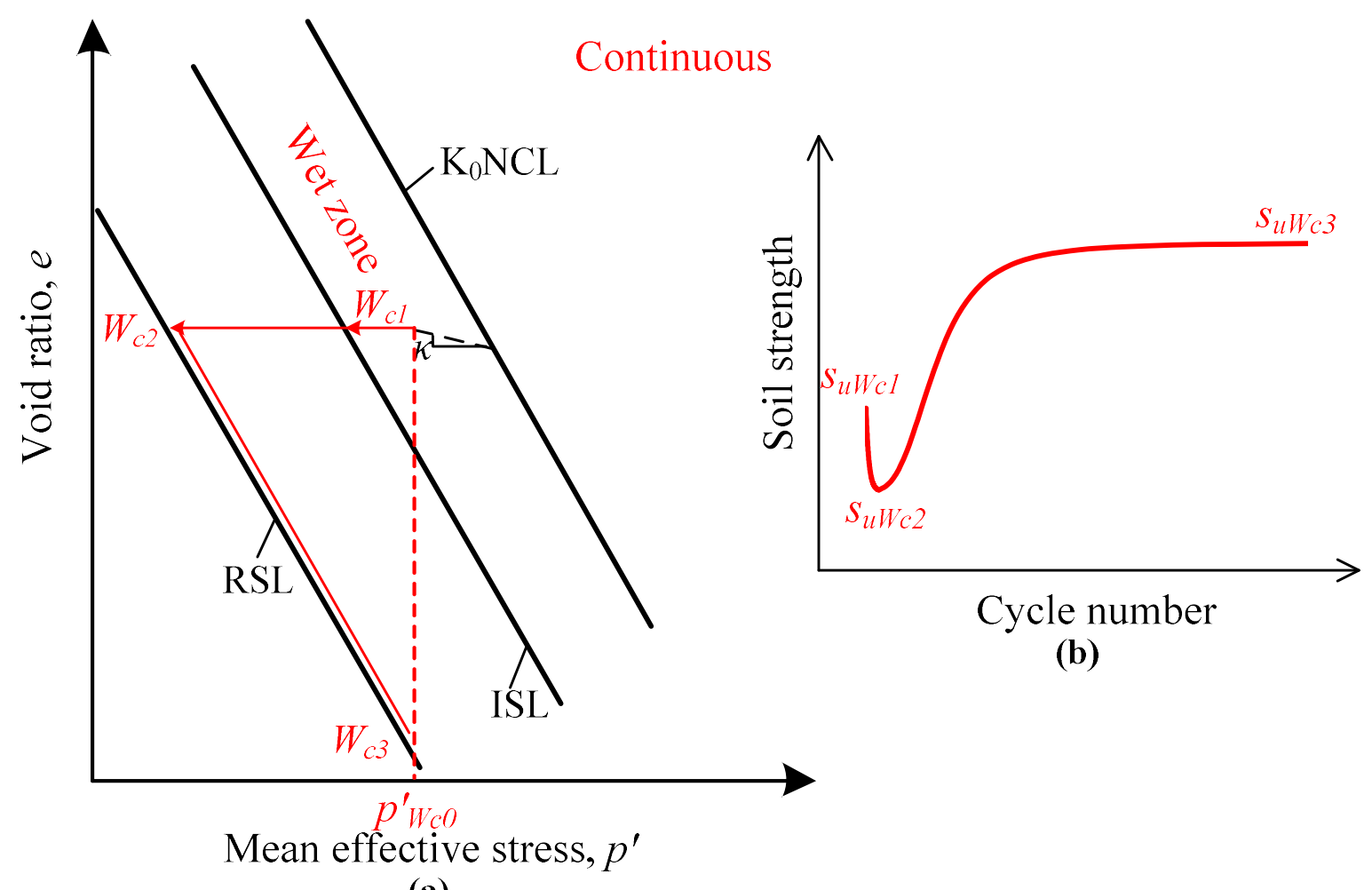

(a)

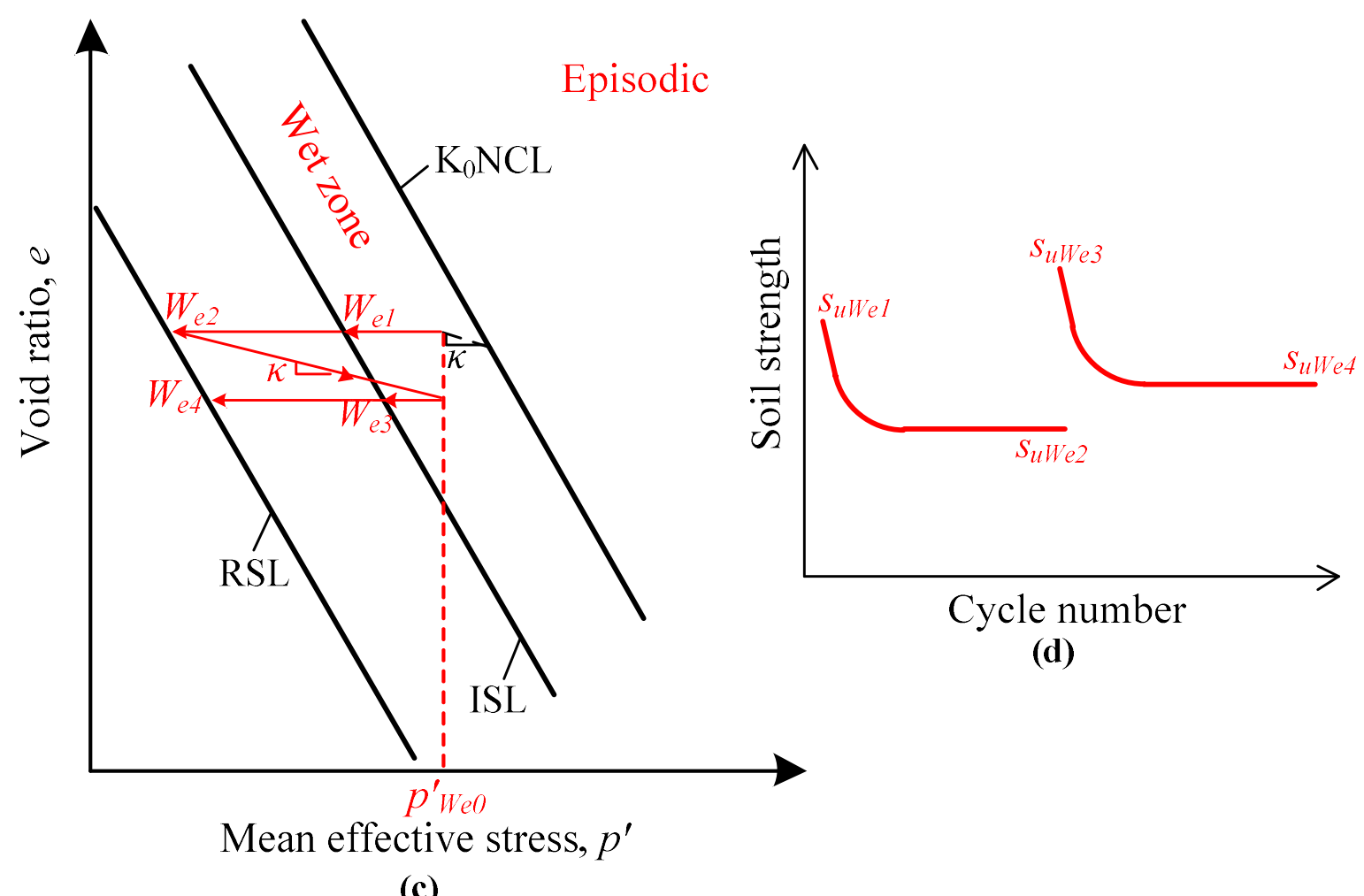

Figure 6. Stress path and strength evolution for soils in the wet zone 


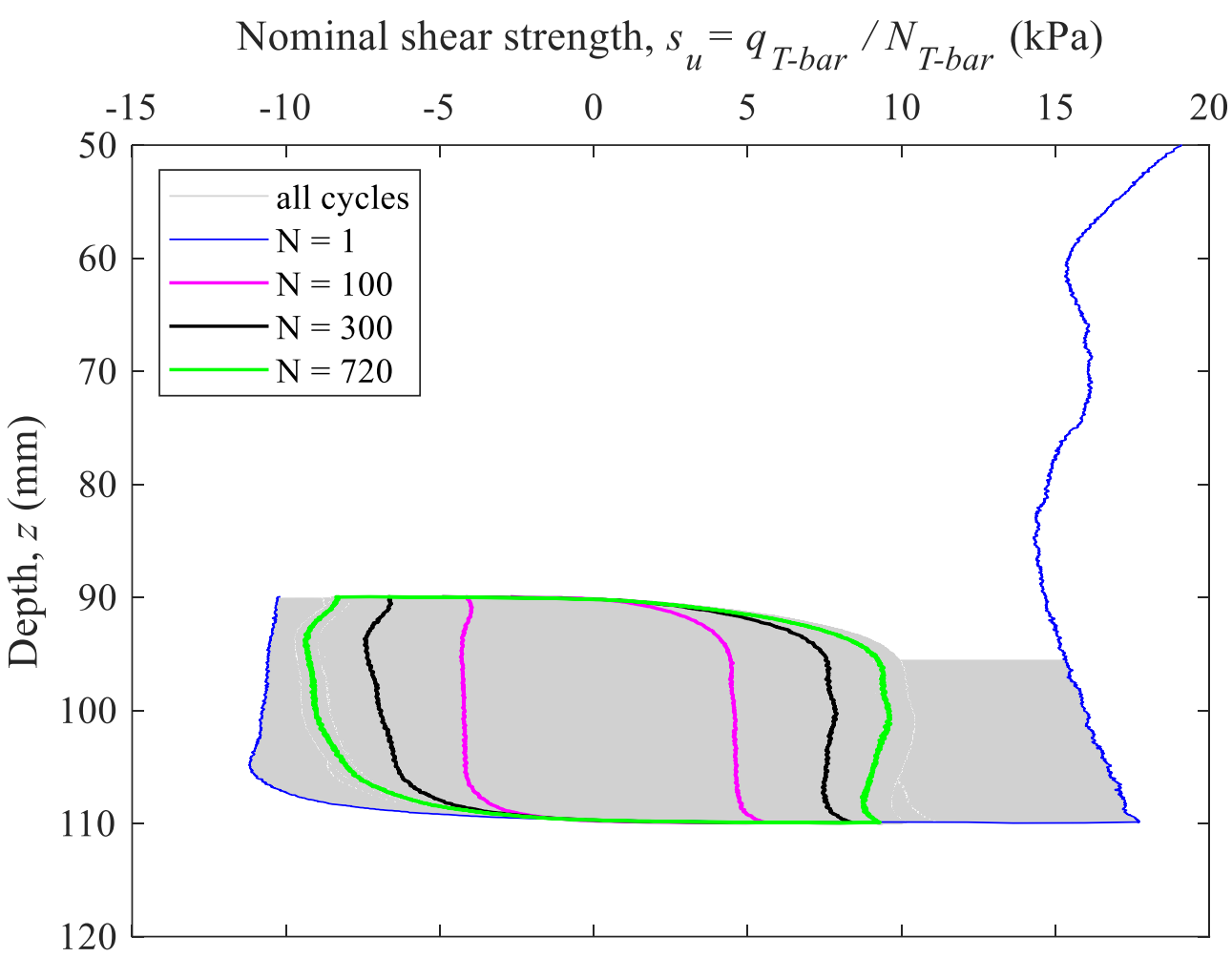

(a)

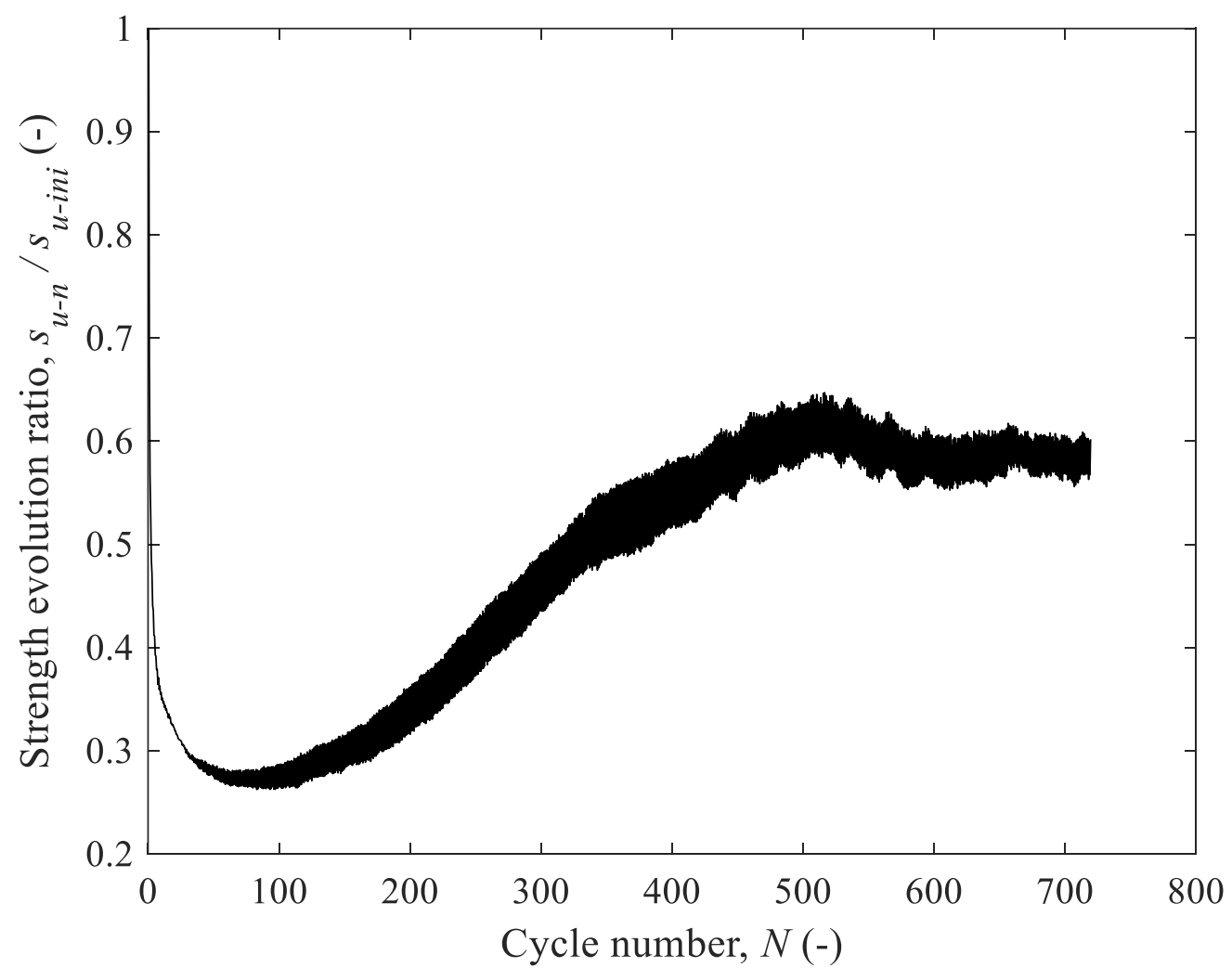

(b)

Figure 7. Continuous cyclic T-bar test results in kaolin sample (test DC2-K; OCR = 10), assuming $N_{T-\text { bar }}=10.5$ : (a) T-bar overall strength profile; (b) evolution of strength at the mid-cycle depth 


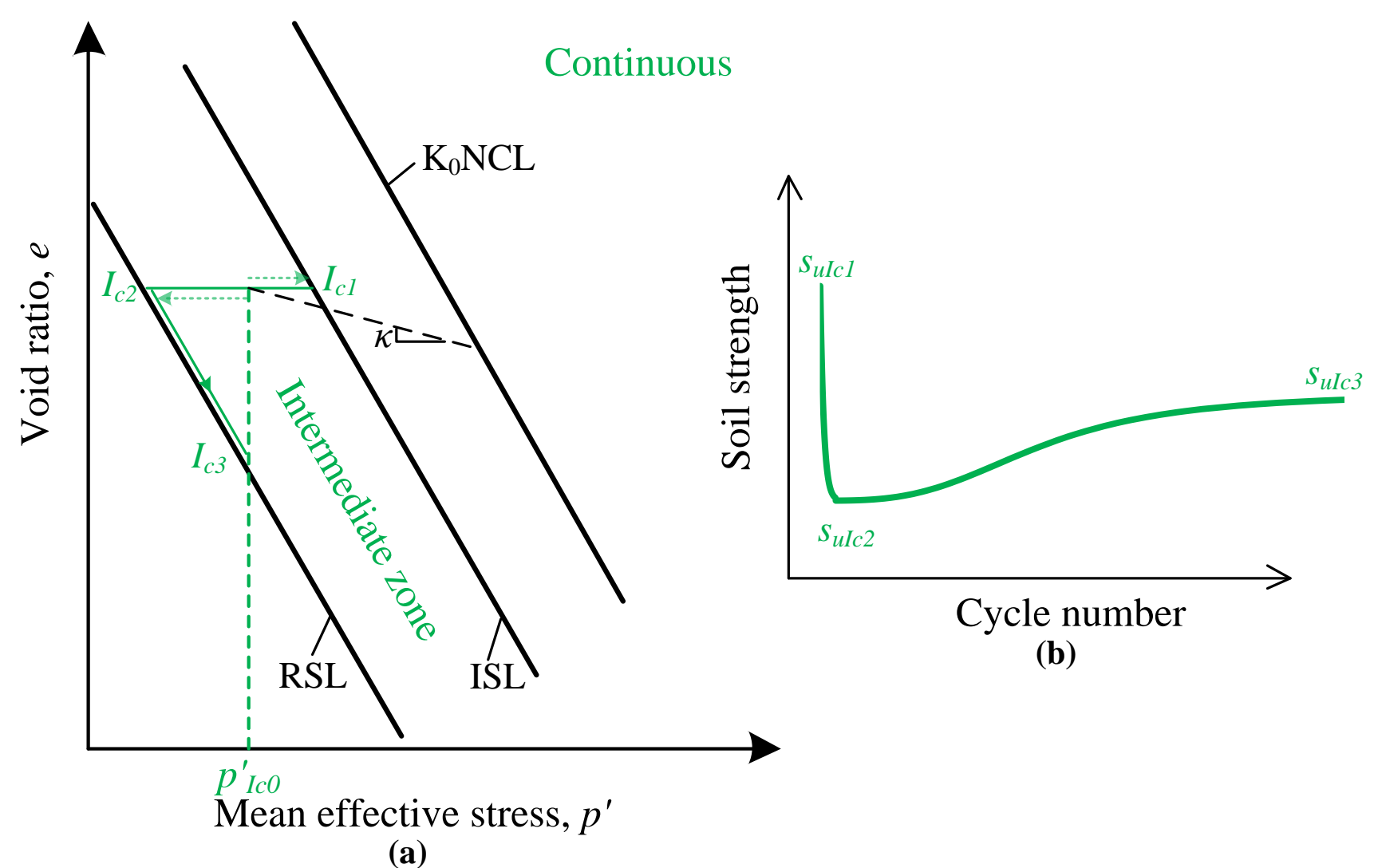

Figure 8. Stress path and strength evolution for soils in the intermediate zone 


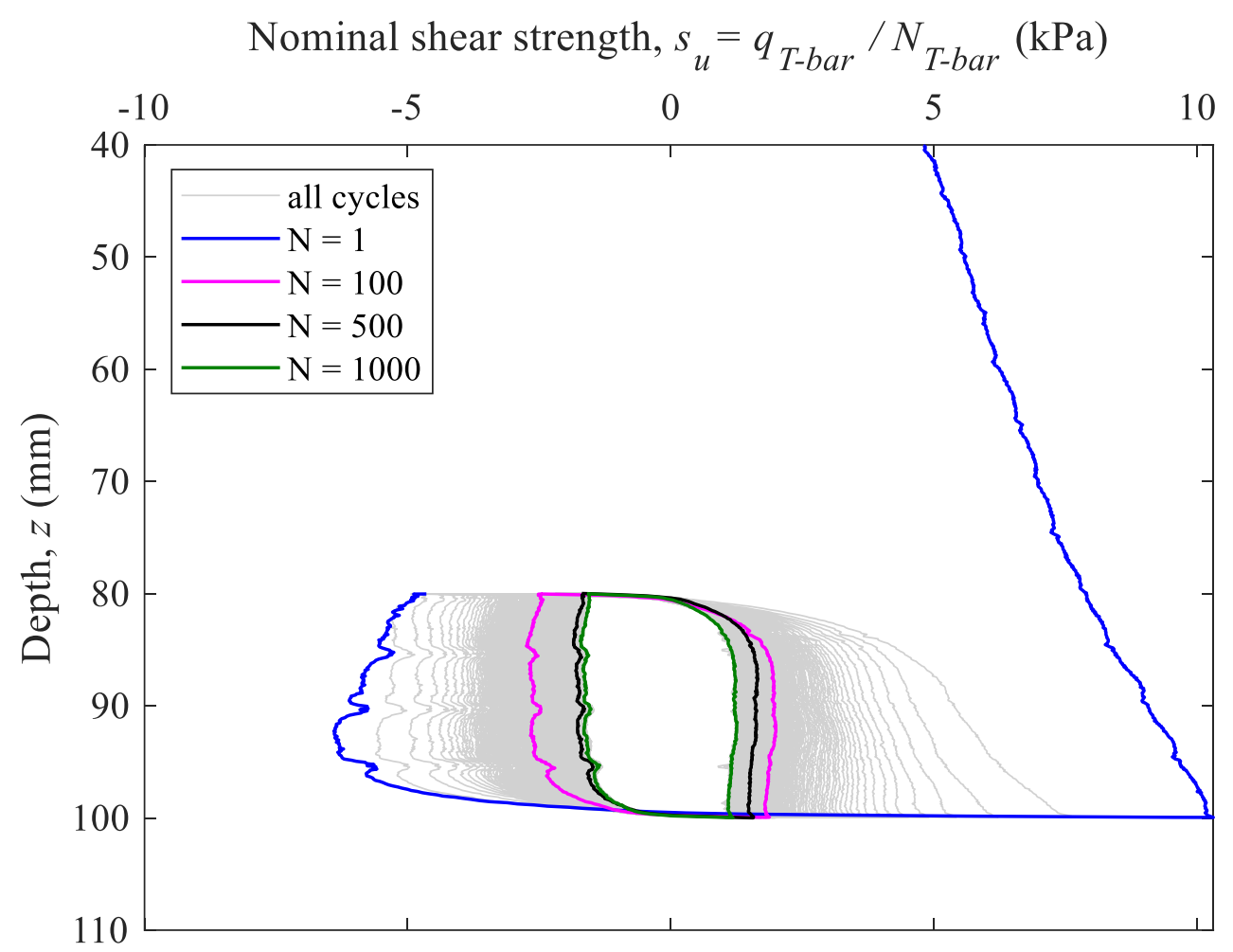

(a)

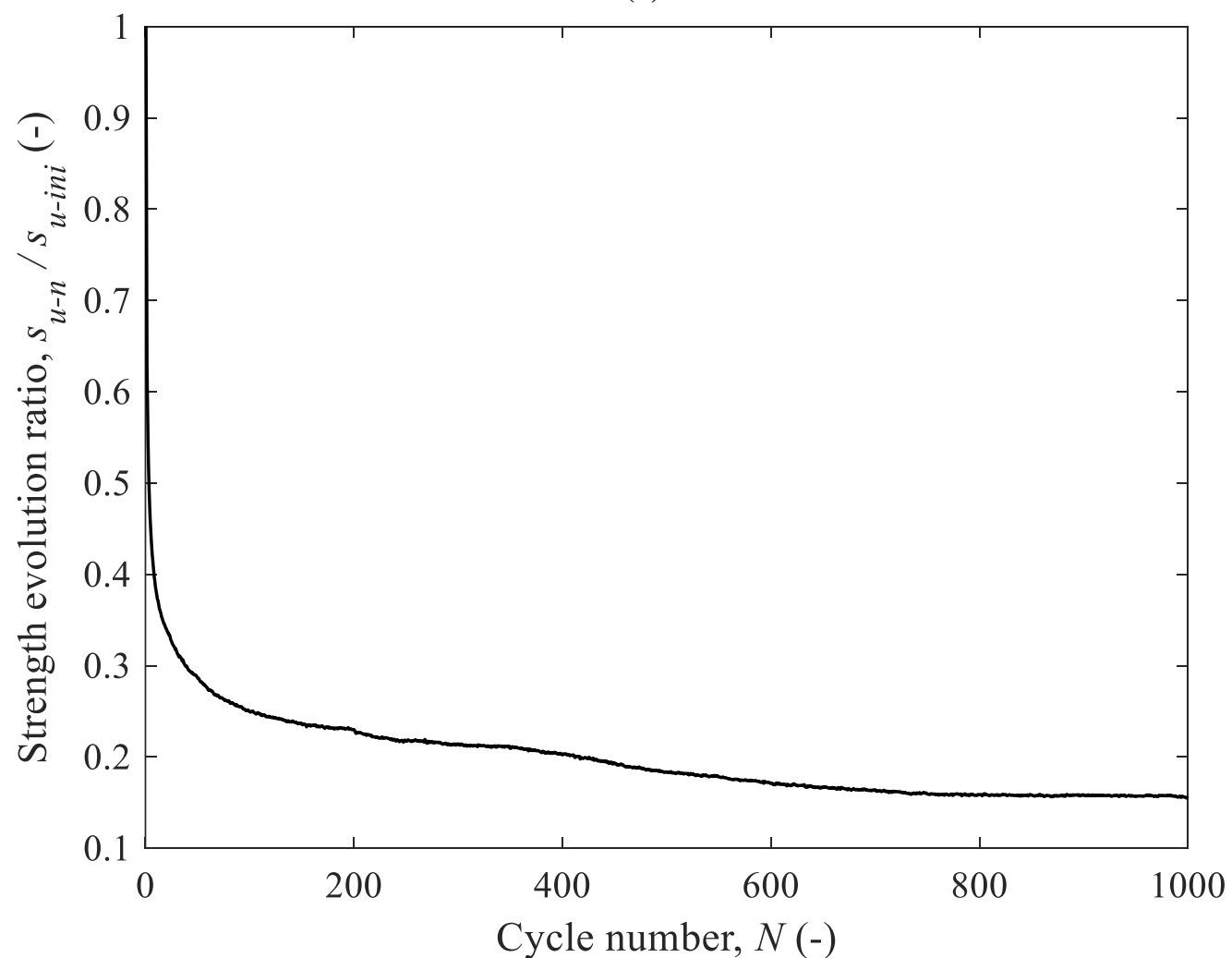

(b)

Figure 9. Continuous cyclic T-bar test results in kaolin sample (test DC3-K; OCR = 200), assuming $N_{T-\text { bar }}=10.5$ : (a) T-bar overall strength profile; (b) evolution of strength at the mid-cycle depth 


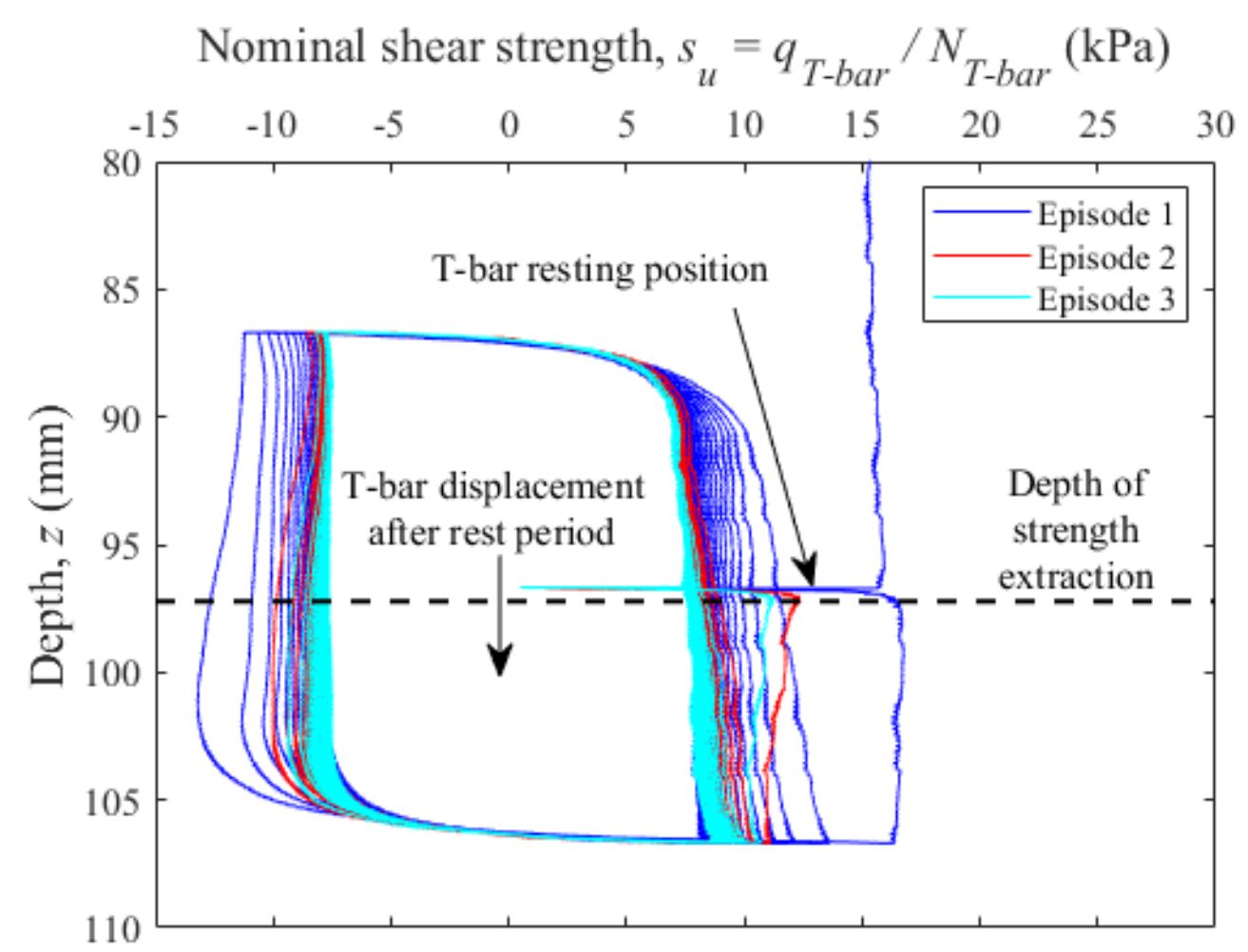

(a)

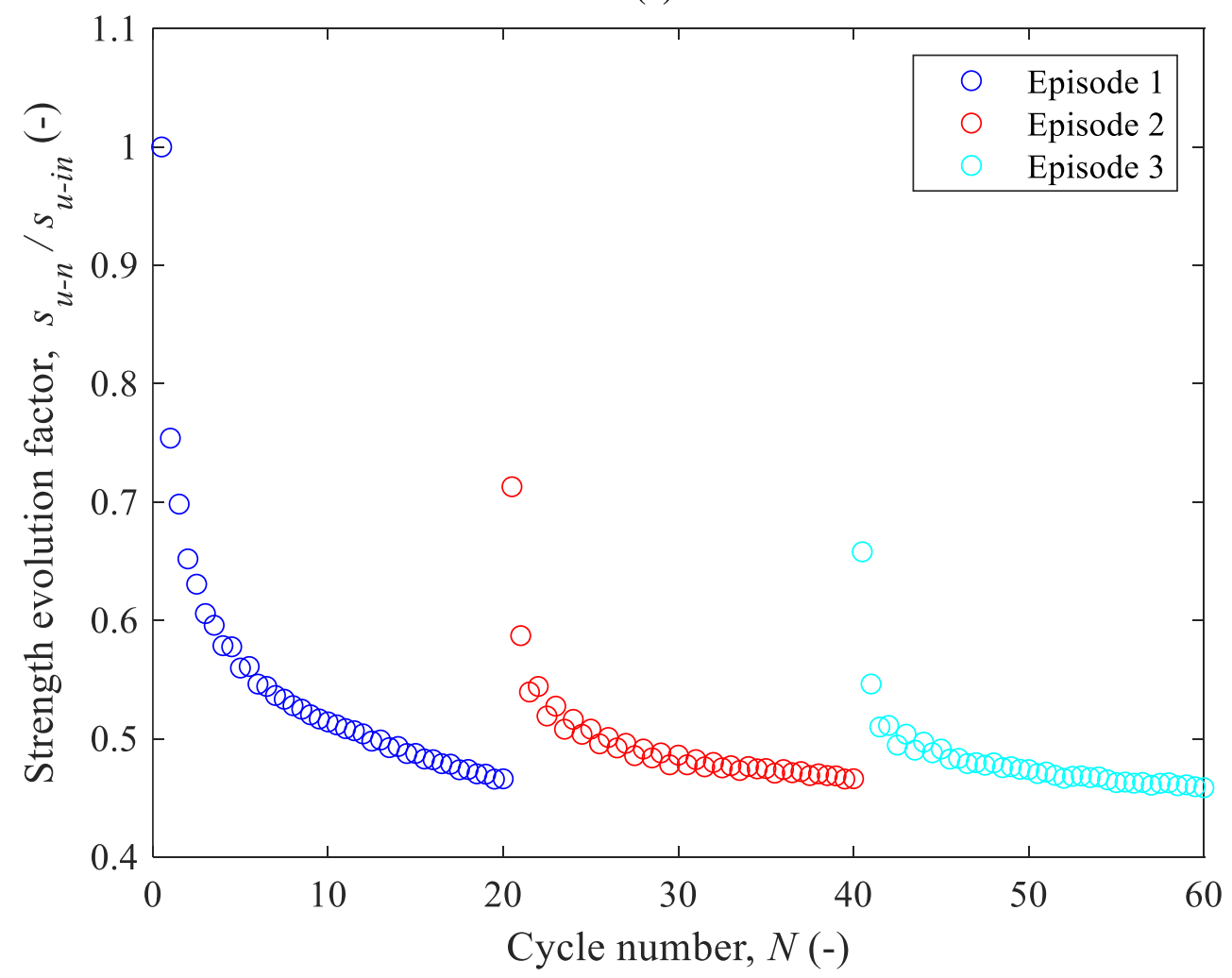

(b)

Figure 10. Episodic cyclic T-bar tests in sample GoM2 (test DC-GoM2; OCR = 10), assuming $N_{T-b a r}=$ 10.5: (a) T-bar overall strength profile; (b) evolution of strength immediately below the T-bar resting location 

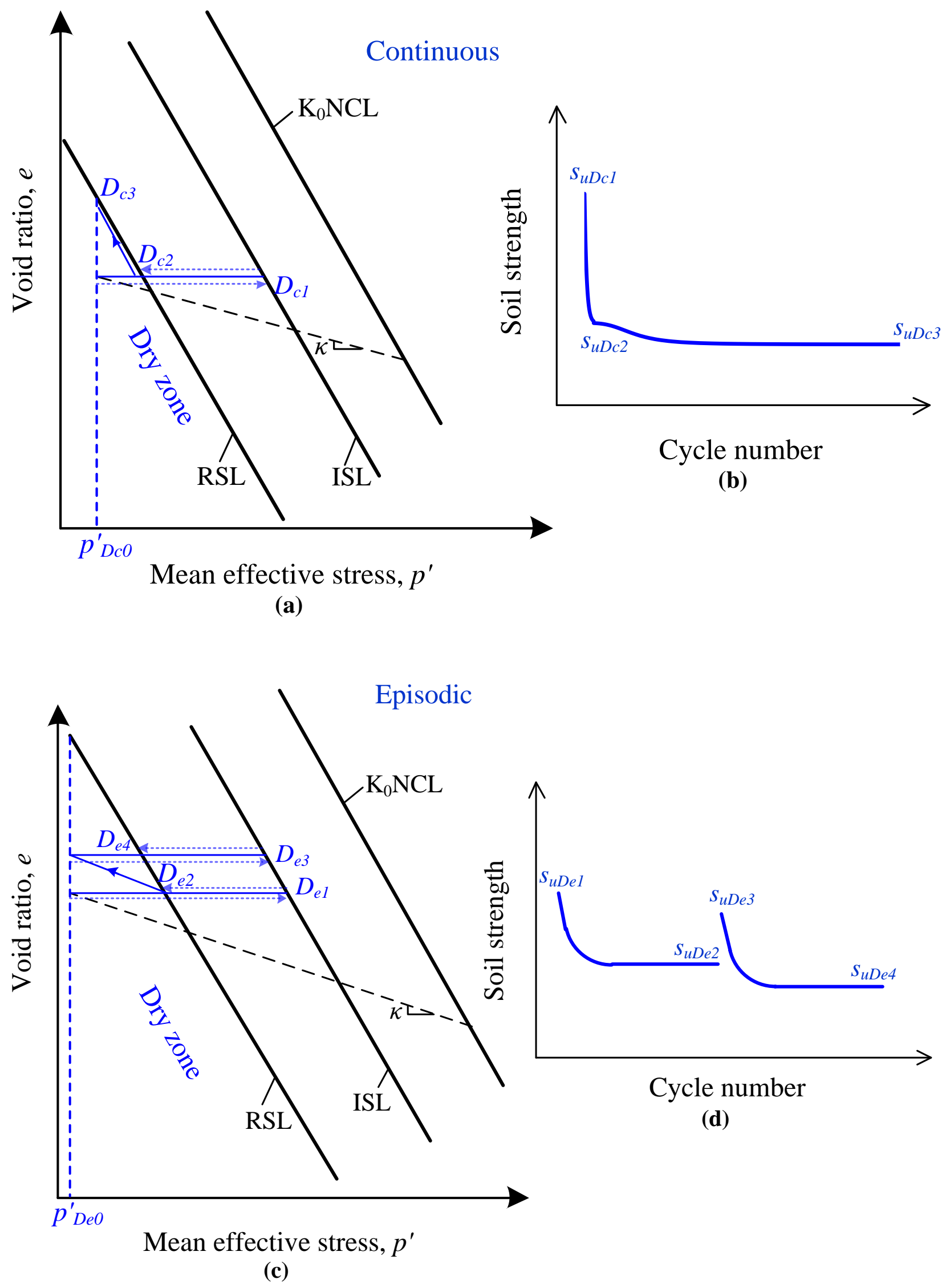

Figure 11. Stress path and strength evolution for soils in the dry zone 


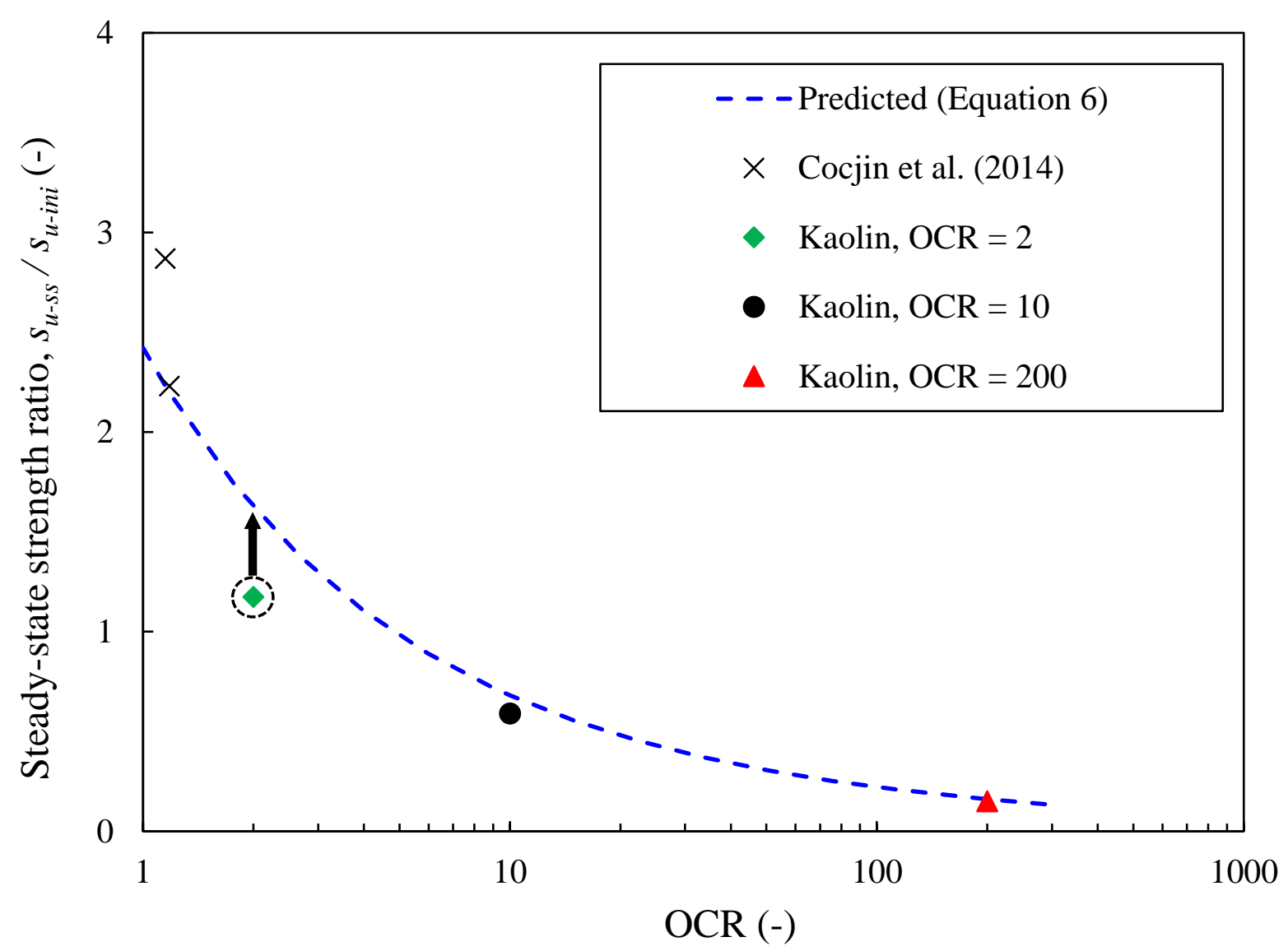

Figure 12. Steady-state $s_{u-s s} / s_{u-i n}$ ratio 


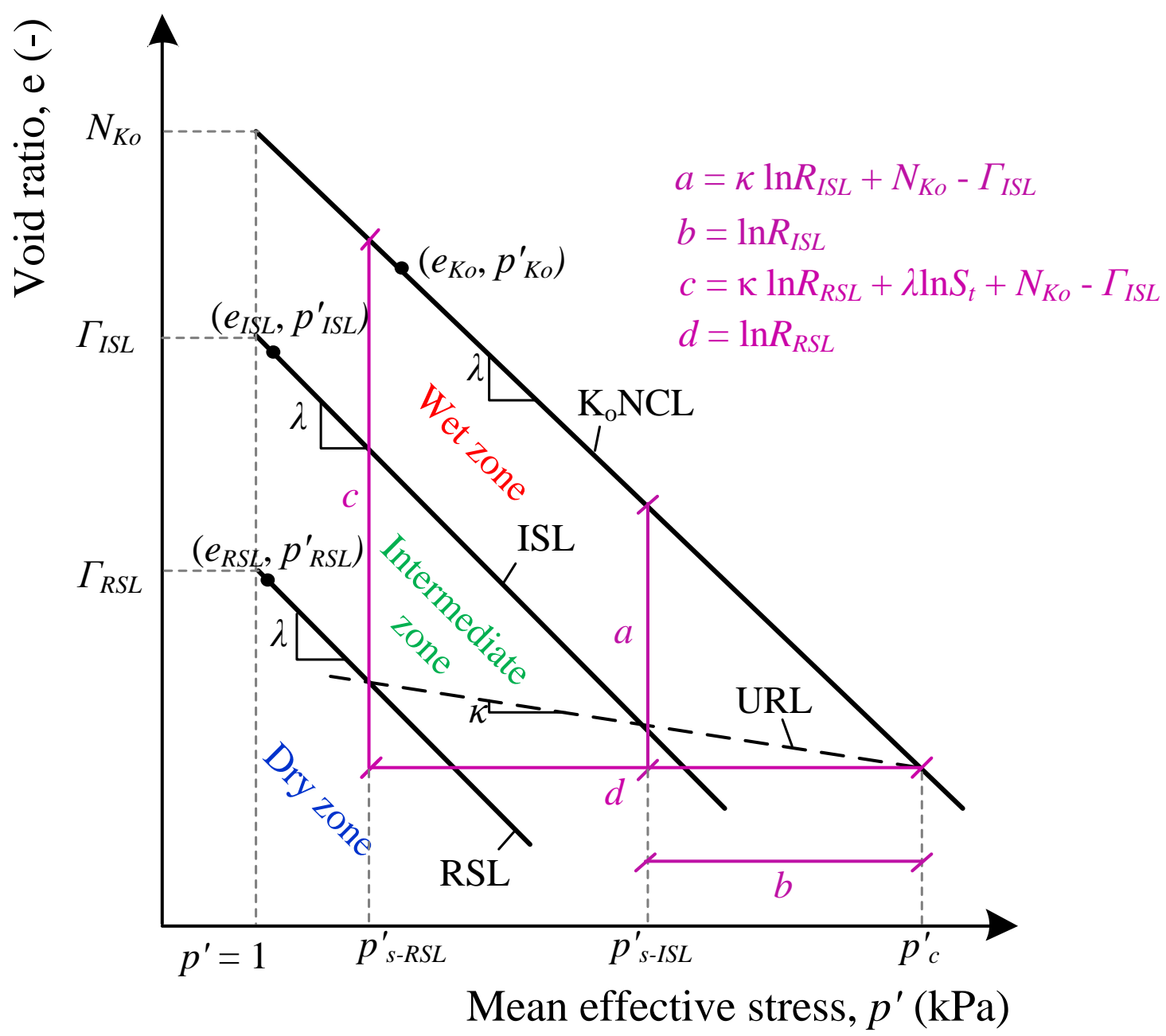

Figure A1. Soil state framework in $e-p^{\prime}$ space 